Draft VERSION JULY 11, 2018

Typeset using $\mathrm{LAT}_{\mathrm{E}} \mathrm{X}$ modern style in AASTeX61

\title{
THE LARGE-SCALE INTERSTELLAR MEDIUM OF SS 433/W50 REVISITED
}

\author{
Yang Su, ${ }^{1}$ Xin Zhou, ${ }^{1}$ Ji Yang, ${ }^{1}$ Yang Chen,,${ }^{2,3}$ Xuepeng Chen, ${ }^{1}$ And \\ SHAOBO ZHANG ${ }^{1}$
} ${ }^{1}$ Purple Mountain Observatory and Key Laboratory of Radio Astronomy, Chinese Academy of
Sciences, Nanjing 210034, China
${ }^{2}$ Department of Astronomy, Nanjing University, Nanjing 210023, China
${ }^{3}$ Key Laboratory of Modern Astronomy and Astrophysics, Nanjing University, Ministry of Education,
Nanjing 210093, China

\begin{abstract}
With new high-resolution $\mathrm{CO}$ and $\mathrm{H}$ I data, we revisited the large-scale interstellar medium (ISM) environment toward the SS 433/W50 system. We find that two interesting molecular cloud (MC) concentrations, G39.315-1.155 and G40.331-4.302, are well aligned along the precession cone of SS 433 within a smaller opening angle of $\sim \pm 7^{\circ}$. The kinematic features of the two MCs at $\sim 73-84 \mathrm{~km} \mathrm{~s}^{-1}$, as well as those of the corresponding atomic-gas counterparts, are consistent with the kinematic characteristics of SS 433. That is, the receding gas from SS 433 jet is probably responsible for the redshifted feature of G39.315-1.155 near the Galactic plane and the approaching one may power the blueshifted gas of G40.331-4.302 toward the observer. Moreover, the $\mathrm{H}$ I emission at $V_{\mathrm{LSR}} \sim 70-90 \mathrm{~km} \mathrm{~s}^{-1}$ displays the morphological resemblance with the radio nebula W50. We suggest that the $V_{\mathrm{LSR}}=77 \pm 5 \mathrm{~km} \mathrm{~s}^{-1}$ gas is physically associated with SS 433/W50, leading to a near kinematic distance of $4.9 \pm 0.4 \mathrm{kpc}$ for the system. The observed gas features, which are located outside the current radio boundaries of W50, are probably the fossil record of jet-ISM interactions at $\sim 10^{5}$ years ago. The energetic jets of the unique microquasar have profound effects on its ISM environment, which may facilitate the formation of molecular gas on the timescale of $\lesssim 0.1$ Myr for the ram pressure of $\sim 2 \times 10^{6} \mathrm{~K} \mathrm{~cm}^{-3}$.
\end{abstract}

Keywords: ISM: individual objects (SS 433/W50) - ISM: kinematics and dynamics - ISM: jets and outflows - supernova remnants

Corresponding author: Yang Su yangsu@pmo.ac.cn 


\section{INTRODUCTION}

The radio nebula W50, also known as supernova remnant (SNR) G39.7-2.0 (e.g., Green 2017), has a large angular extent of $\sim 120^{\prime} \times 60^{\prime}$, which surrounds its central bright compact point source of SS 433. SS 433, a close massive binary system, is a Galactic microquasar consisting of a compact object and a massive donor star (e.g., see reviews of Margon 1984; Fabrika 2004).

The well-known and unique object SS 433 has been widely studied in mulitwavelength observations, as well as theoretical analyses and numerical simulations. Most of these studies focused on the properties of the energetic microquasar itself. On the other hand, many works were also concentrated on the large-scale environment of the unusual system (e.g., radio continuum studies in Geldzahler et al. 1980; Downes et al. 1981, 1986; Elston \& Baum 1987; Dubner et al. 1998; Gao et al. 2011; Broderick et al. 2018; IR studies in Band 1987; Wang et al. 1990; Mirabel et al. 1996; optical studies in van den Bergh 1980; Zealey et al. 1980; Mazeh et al. 1983; Boumis et al. 2007; Abolmasov et al. 2010; X-ray studies in Watson et al. 1983; Yamauchi et al. 1994; Brinkmann et al. 1996; Safi-Harb \& Ögelman 1997; Safi-Harb \& Petre 1999; Brinkmann et al. 2007; the multi-wavelength studies in Moldowan et al. 2005; $\mathrm{HI}$ and $\mathrm{CO}$ gas studies in Lockman et al. 2007; Yamamoto et al. 2008; and the very recent magnetic fields and ionized gas studies in Farnes et al. 2017).

Among the ample scope on the SS 433/W50 system, the interaction between SS 433/W50 and its surrounding interstellar medium (ISM) is a worthy topic for further studies. The prominent phenomenon of the system is that the elongation of W50 is exactly along the axis of the precession cone of the SS 433 jets. The extension of the relativistic jets of SS 433 (and/or jet counterparts in multiwavelength, e.g., $\mathrm{X}$-ray, optical, and radio emission) has an orientation coincident with that of W50 nebula, suggesting the physical connection between them. But for all this, the origin of them is still uncertain and debated (e.g., more details in Farnes et al. 2017).

In this paper, we use the Milky Way Imaging Scroll Painting (MWISP ${ }^{1}$ ) CO data and the complementary H I data from the Green Bank Telescope (GBT) $100 \mathrm{~m}$ and Arecibo Observatory $305 \mathrm{~m}$ telescopes to investigate the molecular and atomic gas properties of W50. Thanks to the Galactic CO and H I surveys with the highdynamical range, we can gain much insight into the ISM environment of SS 433/W50 from the combined high-quality molecular and atomic line observations. Especially, the high-resolution (in spatial and velocity) $\mathrm{CO}$ and $\mathrm{H}$ I data allow us to investigate the gas properties and the kinematic features of the surrounding ISM of SS 433/W50 from the large-scale structures of several degrees to the small-scale features of $\lesssim 1$ arcmin.

\footnotetext{
${ }^{1}$ http://english.dlh.pmo.cas.cn/ic/
} 
Throughout this paper, we use Galactic coordinates to identify directions on the sky, which is convenient to analyze the structure of the gas on a large scale, e.g., several degrees. In particular, the well-known western and eastern jets/lobes of SS 433/W50 in Equatorial coordinates are described as the northwestern jet/lobe (the receding one toward the Galactic plane) and the southeastern jet/lobe (the approaching one away from the plane) in Galactic coordinates (see the radio morphology of W50 in Figure 1 and the red and blue lines in Figure 4), respectively.

\section{OBSERVATIONS AND DATA}

\subsection{CO data}

The CO data used in this work are part of the MWISP project, which is a large, unbiased, and high-sensitivity $\mathrm{CO}$ survey toward the Galactic plane for the region of $l=\left[-10^{\circ}, 250^{\circ}\right]$ and $b=\left[-5^{\circ}, 5^{\circ}\right] .{ }^{12} \mathrm{CO}(J=1-0),{ }^{13} \mathrm{CO}(J=1-0)$, and $\mathrm{C}^{18} \mathrm{O}(J=1-0)$ lines were observed simultaneously using the $13.7 \mathrm{~m}$ millimeter-wavelength telescope located at Delingha in China. A $3 \times 3$ beam array (Shan et al. 2012) was designed to monitor nine positions at once, increasing the mapping speed by roughly an order of magnitude. The total bandwidth is $1 \mathrm{GHz}$ and the half-power beamwidth (HPBW) of the telescope is about $50^{\prime \prime}$ for the three lines. The typical rms noise level is about 0.5 $\mathrm{K}$ for ${ }^{12} \mathrm{CO}(J=1-0)$ at the channel width of $0.16 \mathrm{~km} \mathrm{~s}^{-1}$ and $0.3 \mathrm{~K}$ for ${ }^{13} \mathrm{CO}(J=1-0)$ and $\mathrm{C}^{18} \mathrm{O}(J=1-0)$ at $0.17 \mathrm{~km} \mathrm{~s}^{-1}$. The details of the $13.7 \mathrm{~m}$ telescope can be found from the status report ${ }^{2}$.

The observing strategy, the instrument, and the quality of the $\mathrm{CO}$ observations were described in our recent paper (see Su et al. 2017b). Briefly, Each $30^{\prime} \times 30^{\prime}$ map was covered with position-switch On-The-Fly mode at least twice in scanning direction along Galactic longitude and latitude to minimize the fluctuation of noise. The scan speed was $50^{\prime \prime} \mathrm{s}^{-1}$ ( or $75^{\prime \prime} \mathrm{s}^{-1}$ ) with a dump time of $0.3 \mathrm{~s}$ (or $0.2 \mathrm{~s}$ ). The sampling interval was $15^{\prime \prime}$ and the spacing between scan rows was $10^{\prime \prime}$, fulfilling the requirements for oversampling of the $50^{\prime \prime}$ beam of $13.7 \mathrm{~m}$ telescope. After the first order (linear) baseline fitting and mosaicing the image, the final cube data were constructed with a grid spacing of 30". All data were reduced using the GILDAS software $^{3}$. The SS 433/W50 region was completely mapped during 2012 to 2015 (see Su et al. 2016).

\subsection{H I data and radio continuum emission}

To compare with the large-scale molecular gas, we used the $100 \mathrm{~m}$ GBT $21 \mathrm{~cm}$ emission line of H I (see the details in Lockman et al. 2007) as a tracer of the neutral atomic gas. The final GBT H I data were gridded to 3.5 with a velocity separation of $1.03 \mathrm{~km} \mathrm{~s}^{-1}$. The further Galactic ALFA H I (GALFA; Peek et al. 2011) survey data, which were done with the Arecibo Observatory $305 \mathrm{~m}$ telescope, were also

${ }^{2}$ http://www.radioast.nsdc.cn:81/mwisp.php

${ }^{3}$ http://ascl.net/1305.010 or http://www.iram.fr/IRAMFR/GILDAS 
investigated. The GALFA H I cube data have a grid spacing of 1'.0 and a velocity channel separation of $0.184 \mathrm{~km} \mathrm{~s}^{-1}$. Typical noise levels are $0.1 \mathrm{~K} \mathrm{rms}$ of brightness temperature in an integrated $1 \mathrm{~km} \mathrm{~s}^{-1}$ channel for both the GBT and GALFA H I data. Finally, the radio continuum emission of SS 433/W50 was from the Effelsberg $11 \mathrm{~cm}$ survey (Reich et al. 1990).

\section{THE MOLECULAR AND ATOMIC GAS TOWARD SS 433/W50}

Several works had been done to investigate the gas environment toward the SS 433/W50 system (e.g., Huang et al. 1983; Dubner et al. 1998; Durouchoux et al. 2000; Lockman et al. 2007; Yamamoto et al. 2008). However, conclusions from these studies, especially on the kinematic distance to SS 433/W50 (e.g., 2.2-5.5 kpc), are not consistent with each other. In these papers, the local standard of rest (LSR) velocity of the gas was used to determine the distance of the system based on $\mathrm{HI}$ absorption and/or $\mathrm{CO}+\mathrm{H}$ I associations with W50 nebula. As an association between SS 433/W50 and its surrounding gas was established, the kinematic distance of them at a certain LSR velocity can be determined from the Galactic rotation curve model. Therefore, the key point to the argument is which of the gas components along the line of sight (LOS) is actually associated with SS 433/W50.

A detailed analysis of the combined molecular and atomic gas toward the direction could shed light on the connection between the SS 433/W50 system and its surrounding ISM. The new high-resolution and high-sensitivity CO observations, together with the complementary $\mathrm{H}$ I data, may also provide us a good opportunity to investigate interactions of the system with its ambient gas on a large scale.

Generally, a large amount of molecular gas traced by CO emission, as well as the complicated atomic gas traced by H I data, is seen in the region of SS 433/W50. For the convenience of discussion, the gas toward SS 433/W50 was divided into four parts

according to different velocities of $V_{\mathrm{LSR}} \lesssim 0 \mathrm{~km} \mathrm{~s}^{-1}, V_{\mathrm{LSR}}=0-20 \mathrm{~km} \mathrm{~s}^{-1}, V_{\mathrm{LSR}}=20-$ $70 \mathrm{~km} \mathrm{~s}^{-1}$, and $V_{\mathrm{LSR}} \gtrsim 70 \mathrm{~km} \mathrm{~s}^{-1}$. Position-velocity (PV) diagrams across the radio nebula W50 (e.g., see yellow arrows that are roughly perpendicular to the precession axis of SS 433 in Figure 1) were made in Figure 2 for subsequent analysis.

\subsection{Gas at $\lesssim 0 \mathrm{~km} \mathrm{~s}^{-1}$}

The gas with a negative velocity is believed to lie beyond the solar circle in the direction. A little molecular gas with $V_{\mathrm{LSR}} \lesssim 0 \mathrm{~km} \mathrm{~s}^{-1}$ was detected near the radio boundary of SS 433/W50 in the MWISP CO survey. These molecular clouds (MCs) are located within the distant Outer Arm (e.g., MWISP G039.923-00.655 at $V_{\mathrm{LSR}} \sim-31 \mathrm{~km} \mathrm{~s}^{-1}$, Su et al. 2016) or the Extreme Outer Galaxy region (e.g., MWISP G039.175-01.425 and MWISP G039.225-01.524 at $V_{\mathrm{LSR}} \sim-55 \mathrm{~km} \mathrm{~s}^{-1}$, Sun et al. 2017). We confirm that the MCs with negative velocities are not related to SS 433/W50 due to their large kinematic distances, e.g., $d>13 \mathrm{kpc}$.

One may wonder whether these MCs were perturbed or accelerated by the highvelocity gas from SS 433 jet. However, this possibility is excluded due to lacking 
any kinematic signatures (e.g., prominent line broadenings or asymmetric line profiles) and/or velocity gradients within the precession cone of SS 433 for the gas with negative velocities. After checking the channel maps of $\mathrm{H}$ I data, we cannot find any large-scale morphological correspondence between the atomic gas and the extended radio emission of $\mathrm{W} 50$. There seems to be no significant accelerated gas toward us

in the velocity range of $V_{\mathrm{LSR}} \lesssim 0 \mathrm{~km} \mathrm{~s}^{-1}$, which is in agreement with the analysis of Lockman et al. (2007). We will mainly focus on the gas within the solar circle $\left(V_{\mathrm{LSR}} \gtrsim 0 \mathrm{~km} \mathrm{~s}^{-1}\right)$ in the subsequent sections.

\subsection{Gas at 0-20 $\mathrm{km} \mathrm{s}^{-1}$}

In the velocity range, most of the $\mathrm{CO}$ emission, which is widely distributed in the field of view (FOV), is obvious from the Local Arm because of their large-scale angular sizes in space (e.g., several tens arcmins to several degrees) and their broad distribution along the Galactic latitude (e.g., $b=-5^{\circ} .1$ to $b=+5^{\circ} .1$ ). Briefly, the MCs, which are embedded in diffuse CO emission, display filamentary structures or irregular morphologies on a relatively large scale. Small and faint MCs with various structures can be seen everywhere. Some bright MCs with relatively small angular sizes (e.g., several arcmins to $\lesssim 10$ arcmins), which are mainly concentrated in the Galactic plane of $b \sim-0.4-+0.6$, probably belong to the distant Perseus Arm in the direction. The details of the CO emission in the MWISP survey will be presented elsewhere.

According to the PV diagrams (Figure 2) across the W50 nebula, we do not detect any protruding velocity structures that are often used to trace shock-MC interactions (e.g., Su et al. 2014a,b, 2017a). Further CO spectral analysis does not reveal any striking line diagnostics near and within the radio boundary of the nebula. It indicates that the molecular gas in the interval of $0-20 \mathrm{~km} \mathrm{~s}^{-1}$ is relatively quiescent in the projected area of W50. Wherever the molecular gas at such the velocity interval is from, there is little morphological correspondence between the molecular gas and the W50 nebula. The H I emission, which is more diffuse and extended than CO emission, do not show any prominent connection with W50 either. We conclude that the gas in the velocity range is not related to SS 433/W50.

\subsection{Gas at 20-70 $\mathrm{km} \mathrm{s}^{-1}$}

\subsubsection{The 170 pe long MC filament G40.82-1.41 at 29-34 $\mathrm{km} \mathrm{s}^{-1}$}

According to the CO intensity map in the interval of $20-40 \mathrm{~km} \mathrm{~s}^{-1}$ (Figure 1), a giant molecular filament (GMF), which is named as G40.82-1.41 from its geometric center of the $\mathrm{CO}$ emission, is revealed to extend about 5 degrees from $(l=42.9, b=-0.1)$ to $(l=38.7, b=-2.7)$. The distance to the GMF is estimated to be $1.6-2.0 \mathrm{kpc}$ (e.g., the 3D extinction map from Green et al. 2015) or $\sim 1.8-2.1 \mathrm{kpc}$ from the CO peak velocities of $V_{\mathrm{LSR}} \sim 29-34 \mathrm{~km} \mathrm{~s}^{-1}$ (the near kinematic distance from the A5 model of Reid et al. 2014). The length of the GMF is thus about $170 \mathrm{pc}$ at a distance of $\sim 2.0 \mathrm{kpc}$. 
The GMF G40.82-1.41 is a clustering of tens of small filaments with somewhat different LSR velocities. These small filaments consisting of multiple components exhibit complicated structures. Many of them are oriented parallel to the long axis of the GMF and appear to be twisted between each other. The southwestern part of the GMF across the radio nebula W50 was suggested to have a connection with the SNR W50 (see Figure 3 and discussions in Huang et al. 1983). Based on PV diagrams (e.g., LPV2 and LPV3 in Figure 2), however, we cannot find significant kinematic features of shock-MCs interactions near and within the boundary of W50 nebula. Any asymmetric CO line profiles, which can be seen everywhere both within and outside the W50 nebula, are obviously attributed to overlapping components at somewhat different velocities.

\subsubsection{The MCs along the precession cone of SS 433 and the corresponding HI gas at $\sim 40-60 \mathrm{~km} \mathrm{~s}^{-1}$}

Several interesting MCs seem roughly aligned along the precession cone of SS 433, which was suggested to be the evidence of SS 433/W50-ISM interactions (see Figures 1-4 and discussions in Yamamoto et al. 2008). Combined with the radio continuum and H I studies by Dubner et al. (1998), Yamamoto et al. (2008) then placed these MCs (the northern MCs of N1-N4 at $V_{\mathrm{LSR}} \sim 53 \mathrm{~km} \mathrm{~s}^{-1}$ and the southern MCs of S1-S6 at $V_{\mathrm{LSR}} \sim 43 \mathrm{~km} \mathrm{~s}^{-1}$, see Table 1 in their paper) and the associated SS 433 at a near kinematic distance of $\sim 3 \mathrm{kpc}$.

Figure 3 shows the WISE $22 \mu \mathrm{m}$ emission overlaid with MWISP CO contours (blue, corresponding to MCs S1-S6 in Yamamoto et al. 2008) in the interval of $39-51 \mathrm{~km} \mathrm{~s}^{-1}$ and GALFA H i contours (red) in the interval of $35-48 \mathrm{~km} \mathrm{~s}^{-1}$. Obviously, the bright diffuse IR emission, which displays multiple shell-like or bubble-like features, is coincident well with the gas emission at $V_{\mathrm{LSR}} \sim 43 \mathrm{~km} \mathrm{~s}^{-1}$, suggestive of an association between the IR features and the gas. The near kinematic distance of the gas is about $2.7 \mathrm{kpc}$ (Reid et al. 2014), which is in good agreement with the $2.0-2.5 \mathrm{kpc}$ from the 3D extinction map of Green et al. (2015). The atomic and molecular gas, together with the bright thermal dust association centered at $\left(l \sim 40.4, b \sim-4^{\circ} .3\right)$ with a radius of $\sim 1^{\circ}$, is probably related to the star-forming regions at a distance of $\sim 2.5 \mathrm{kpc}$ (or 190 pc below the Galactic plane). The total mass of the atomic+molecular gas within the region of $\sim 90 \times 90 \mathrm{pc}^{2}$ is $\gtrsim 1 \times 10^{5} M_{\odot}$. It is hard to believe that such amounts of gas can originate from the jet-ISM process of SS 433.

On the other hand, MCs at $50-55 \mathrm{~km} \mathrm{~s}^{-1}$ were suggested to be related to the W50 lobe toward the Galactic plane due to the spatial coincidence between them (Durouchoux et al. 2000; Chaty et al. 2001; Yamamoto et al. 2008). Most of the MCs, which are near the HII region Sh 2-74 and close to the Galactic plane, also have corresponding bright IR emission. We argue that these MCs with prominent ${ }^{13} \mathrm{CO}$ emission are probably associated with $\mathrm{H}$ II region Sh 2-74 and/or belong to the nearby MC complex with active star formation (see Section 3.3.3), which agrees with suggestions from previous studies (e.g., Band \& Gordon 1989; Moldowan et al. 2005). 
At $38-44 \mathrm{~km} \mathrm{~s}^{-1}$, a large $\mathrm{H}$ I void-like feature roughly shows the morphological correspondence with the radio emission of W50 (e.g., see Figure 14 in Lockman et al. 2007). However, the large-scale atomic cavity seen in the integrated map seems to be comprised of several-separated substructure of $\mathrm{H}$ I emission. We cannot find fine morphological agreement between the radio continuum emission of W50 and its nearby atomic gas after analyzing the Arecibo $\mathrm{H}$ I data channel-by-channel. We agree with the conclusion of Lockman et al. (2007) that the gas in the velocity range is not physically associated with SS 433/W50.

\subsubsection{Other interesting $M C s$}

The H II region Sh 2-74 ( $l \sim 39.9, b \sim-1.3)$, which has a large radio size of $\sim 1^{\circ} \times 1^{\circ}$ at a distance of about $3 \mathrm{kpc}$ (e.g., Elston \& Baum 1987; Paladini et al. 2003), lies near the northeastern edge of the W50 nebula (Figure 1). Using MWISP CO data, we find that the molecular gas at $40-55 \mathrm{~km} \mathrm{~s}^{-1}\left(V_{\text {peak }} \sim 48 \mathrm{~km} \mathrm{~s}^{-1}\right.$, which agrees well with the RRL central velocity of $\sim 47.7 \mathrm{~km} \mathrm{~s}^{-1}$ for Sh 2-74, Alves et al. 2012), is physically associated with the $\mathrm{H}$ II region according to the morphological agreement and the corresponding kinematic features between the ionized gas of the H II region and the surrounding MCs (see LPV1 and LPV2 in Figure 2).

An interesting $\mathrm{MC}$ at $\left(l \sim 40.66, b \sim-2.42, V_{\text {peak }} \sim 57 \mathrm{~km} \mathrm{~s}^{-1}\right)$ has broad $\mathrm{CO}$ wings in the velocity range of $45-72 \mathrm{~km} \mathrm{~s}^{-1}$ (see LPV3 in Figure 2), which is very likely associated with the H II region Du 22 centered at $\left(l=40^{\circ} 6567, b=-2^{\circ} .4658\right)$ (Dubout-Crillon 1976). The near kinematic distance of the object and the associated MCs is estimated to be $\sim 3.5 \mathrm{kpc}$. The far one of $\sim 9.0 \mathrm{kpc}$ may be excluded due to the unreasonable scale-height of $\sim 400 \mathrm{pc}$.

Based on the above analysis, we argue that the MCs in the velocity range of 20-70 $\mathrm{km} \mathrm{s}^{-1}$ are accidental superpositions along the LOS and are not associated with the SS 433/W50 system. Both of the very long GMF (see discussions in Huang et al. 1983) at $V_{\mathrm{LSR}} \sim 29-34 \mathrm{~km} \mathrm{~s}^{-1}$ and the gas features at $V_{\mathrm{LSR}} \sim 40-$ $60 \mathrm{~km} \mathrm{~s}^{-1}$ (Dubner et al. 1998; Durouchoux et al. 2000; Yamamoto et al. 2008) appear to be just the foreground gas of SS 433/W50 in the FOV. We will not discuss these MCs any further because the above gas is not related to SS 433/W50 at a distance of $4.9 \mathrm{kpc}$ (see Section 4).

\subsection{Gas at $\gtrsim 70 \mathrm{~km} \mathrm{~s}^{-1}$}

There are only a few CO clouds $\left(V_{\text {LSR }} \gtrsim 70 \mathrm{~km} \mathrm{~s}^{-1}, T_{\text {peak }} \sim 1-2 \mathrm{~K}\right.$, and size $\sim 1-2$ $\left.\operatorname{arcmin}^{2}\right)$ within the radio boundary of the W50 nebula, e.g., $(l=39.225, b=-1.942$, $\left.V_{\text {peak }} \sim 73 \mathrm{~km} \mathrm{~s}^{-1}\right)$ and $\left(l=39.692, b=-2.450, V_{\text {peak }} \sim 74 \mathrm{~km} \mathrm{~s}^{-1}\right)$, indicating that the $\mathrm{CO}$ emission at such velocities is very weak. Interestingly, two intriguing MCs, which are named as G39.315-1.155 and G40.331-4.302, are exactly aligned along the precession axis of SS 433 (see two small boxes in Figure 4).

Figure 5 shows a close-up view of the MCs G39.315-1.155 and G40.331-4.302. The northwestern MC G39.315-1.155 appears to display a coherent structure in the CO 
intensity map of $73-88 \mathrm{~km} \mathrm{~s}^{-1}$, while the southeastern MC G40.331-4.302 consists of two parts, the faint $\mathrm{CO}$ emission in the eastern region (near PVSE lines in the right panel of Figure 5) and the relatively strong emission in the western region (near PVS lines in the figure). Both of the two MC concentrations are along the direction from the northeast to the southwest, which are nearly perpendicular to the precession axis of SS 433 (see red and blue lines in Figure 4).

PV diagrams along selected lines (see black arrows in Figure 5) are shown in Figures 6 and 7, for MCs G39.315-1.155 and G40.331-4.302, respectively. Typical spectra of some regions (see circles in Figure 5) are shown in Figures 8 and 9, respectively. It is interesting to note that MC G39.315-1.155 displays redshifted features while MC G40.331-4.302 displays somewhat blueshifted features in both of the PV diagrams and the typical spectra.

Table 1 lists properties of the atomic and molecular gas toward the main part of G39.315-1.155 and G40.331-4.302. The column density of atomic gas of G40.331-4.302 is calculated via the conversion factor of $1.823 \times$ $10^{18} \mathrm{~cm}^{-2}\left(\mathrm{~K} \mathrm{~km} \mathrm{~s}^{-1}\right)^{-1}$ (Dickey \& Lockman 1990). We cannot calculate the atomicgas properties of G39.315-1.155 due to the strong background H I emission near the Galactic gas plane. The peak temperature of MC G40.331-4.302 is $\sim 3.4 \mathrm{~K}$ and $\sim 0.6 \mathrm{~K}$ for ${ }^{12} \mathrm{CO}$ and ${ }^{13} \mathrm{CO}$, respectively. Note that if we use LTE assumption, an excitation temperature of $10 \mathrm{~K}$, the beam filling factor of $\sim 0.5$, and $N\left(\mathrm{H}_{2}\right) / N\left({ }^{13} \mathrm{CO}\right)$ $\approx 7 \times 10^{5}$, the $\mathrm{H}_{2}$ column density of G40.331-4.302 is about $1.1 \times 10^{21} \mathrm{~cm}^{-2}$, which is somewhat larger than the estimated value from the X-factor method (e.g., the mean CO-to- $\mathrm{H}_{2}$ mass conversion factor of $2 \times 10^{20} \mathrm{~cm}^{-2}\left(\mathrm{~K} \mathrm{~km} \mathrm{~s}^{-1}\right)^{-1}$, Dame et al. 2001; Bolatto et al. 2013). It can be naturally explained because we adopt the peak emission of ${ }^{12} \mathrm{CO}$ and ${ }^{13} \mathrm{CO}$ to represent the property of the whole MC.

For the gas in G40.331-4.302, the velocity dispersion $\left(\sigma_{v}=\frac{\Delta V_{\mathrm{FWHM}}}{2.355}\right)$ is estimated to be $\sim 6 \mathrm{~km} \mathrm{~s}^{-1}$ for the atomic gas and $\sim 1.7 \mathrm{~km} \mathrm{~s}^{-1}$ for the molecular gas, respectively. The mean density of the initial gas environment is on the order of $\sim 1 \mathrm{~cm}^{-3}$, which is a reasonable value estimated from the $V_{\mathrm{LSR}} \sim 70-90 \mathrm{~km} \mathrm{~s}^{-1} \mathrm{HI}$ emission along the precession axis of SS 433. For MC G39.315-1.155, the velocity dispersion of the molecular gas is roughly $3-4 \mathrm{~km} \mathrm{~s}^{-1}$, which is approximately two times larger than that of MC G40.331-4.302. The property indicates that MC G39.315-1.155 is much more turbulent than MC G40.331-4.302.

For G40.331-4.302, the molecular gas is mainly concentrated in a slab with a size of $\sim 32^{\prime} \times 8^{\prime}$ (e.g., Figures 10 and 12). The total mass of G40.331-4.302 should be somewhat larger than the estimated value of $\sim 3 \times 10^{3} M_{\odot}$ in Table 1 because of the unaccounted gas in the northeastern region of the cloud (near PVSE in Figure 5) and the potential $\mathrm{H}_{2}$ gas in CO-dark regions (see Section 6). For G39.315-1.155, the $\mathrm{H}_{2}$ mass within a size of $\sim 17^{\prime} \times 8^{\prime}$ is $\sim 1.1 \times 10^{4} M_{\odot}$.

Obviously, the total mass of G39.315-1.155 is larger than that of G40.331-4.302. The two MCs also have different angular distances from SS 433 (e.g., $\sim 69^{\prime}$ 
for G39.315-1.155 vs. $\sim 129^{\prime}$ for G40.331-4.302) and different extension along the northeast-southwest direction (e.g., $\sim 17^{\prime}$ for G39.315-1.155 vs. $\sim 32^{\prime}$ for G40.331-4.302). Actually, the northwestern radio lobe of W50 is famously shorter and brighter than the southeastern one, which is widely attributed to the denser ambient medium close to the Galactic plane (e.g., Dubner et al. 1998; Lockman et al. 2007; Goodall et al. 2011a). The two MCs features presented here can also be explained by the same reason of the denser environment near the Galactic plane, if these MCs are governed by the SS 433 jets/outflows (see Sections 4-6).

The extended and fragmented $\mathrm{CO}$ gas of the two MC concentrations is well confined to a cone with a small angular extent of $\left(\frac{17}{69} \times \frac{180}{\pi}\right)_{\mathrm{G} 39.315} \sim\left(\frac{32}{129} \times \frac{180}{\pi}\right)_{\mathrm{G} 40.331} \approx 14^{\circ}$ with respect to SS 433 . We note that such the angular extent of the molecular gas is comparable to the result from the current X-ray jet of SS 433 (e.g., $\sim 18^{\circ}$, also seen from SS 433, Brinkmann et al. 2007).

To search for possible evidence of the W50-ISM association, we constructed four integrated H I maps in the intervals of 85-88, 82-85, 79-82, and 76-79 $\mathrm{km} \mathrm{s}^{-1}$ (Figure 11). Several $\mathrm{H}$ I features at the velocity range are indeed found to be positionally coincident with the bright radio shell of W50 in the FOV. Firstly, the H I maps exhibit a cavity-like structure near the Galactic plane, which coincides with the radio morphology of the W50 nebula (also see the static ring in Lockman et al. 2007). Secondly, several features with enhanced HI emission match the bright radio shells of W50 very well (e.g., see panels c and d in Figure 11), Thirdly, these H I enhancements, as well as the bright radio shells, roughly extend along the northwest-southeast direction, which is consistent with the trend of the precession axis of SS 433 (note that the axis is roughly perpendicular to the LOS with an inclination angle of $\approx 80^{\circ}$, Eikenberry et al. 2001).

Finally, the H I gas at $V_{\mathrm{LSR}} \sim 70-90 \mathrm{~km} \mathrm{~s}^{-1}$ is associated with MC G40.331-4.302 (Figures 10-12). The H I concentration near MC G40.331-4.302, which seems to be a part of an expanding $\mathrm{HI}$ shell near the southeastern radio lobe of W50 (or the outermost parts of the approaching gas from the SS 433 jet), was suggested to be related to SS 433/W50 (Lockman et al. 2007). Using the Arecibo H I data, we find that the atomic gas, as well as the corresponding molecular gas at the velocity (e.g., the main part of MC G40.331-4.302), is indeed approaching the observer (Figures 11 and 12), indicative of the association between the gas and the SS 433 jet.

\section{ASSOCIATION OF SS 433/W50 WITH THE $\sim 77 \mathrm{KM} \mathrm{S}^{-1}$ CLOUDS AND THE DISTANCE}

Figure 4 displays that two MC concentrations exactly lie projected on the precession axis of the SS 433 jets. Further analysis shows that the kinematic features of the two MC concentrations are consistent well with the behavior of the SS 433 jets. That is, MC G39.315-1.155 displays the redshifted feature (e.g., especially, PVNW3 and PVNW4 in Figure 6) corresponding to the receding jet of SS 433 and 
MC G40.331-4.302 displays the blueshifted feature (e.g., especially, PVS1 and PVS2 in Figure 7) corresponding to the approaching one.

The blueshifted feature of MC G40.331-4.302 is clearly confirmed from the accompanying atomic gas using the Arecibo H I data (see Figure 12). We speculate that MC G39.315-1.155 also has its accompanying receding atomic gas. However, the very strong background H I emission near the Galactic plane prevents us from obtaining its detailed kinematic information. It is very difficult to discern the possible kinematic feature at levels of several $\mathrm{K}$ from the strong background emission at several tens K. We also emphasize that we cannot identify the disturbed molecular gas in the region closer to the Galactic plane (e.g., $b \gtrsim-1.0$ ) due to the complicated $\mathrm{CO}$ emission (e.g., strong ${ }^{13} \mathrm{CO}$ emission and multiple $\mathrm{CO}$ peaks in the velocity interval of $\left.70-85 \mathrm{~km} \mathrm{~s}^{-1}\right)$.

But despite all this, a H I cavity, which was identified by Lockman et al. (2007) using the GBT observations, does appear toward the W50 radio lobe near the Galactic plane using the new Arecibo data (Figure 11). MC G39.315-1.155 is roughly touching the top wall of the H I cavity (e.g., red contours in panel d of Figure 11). Actually, a patch of atomic gas with enhanced H I emission at $\sim 80-90 \mathrm{~km} \mathrm{~s}^{-1}$ (see the H I gas near red contours in Figure 11) seems to be coincident with the MC, suggesting the possible atomic gas away from the observer.

Additionally, the $\mathrm{H}$ I emission at $\sim 70-90 \mathrm{~km} \mathrm{~s}^{-1}$ displays compelling morphological evidence for an association between W50 and the atomic gas (e.g., Figure 11). Combined the above analysis and results from Section 3.4, all the evidence points to the gas at $\sim 70-90 \mathrm{~km} \mathrm{~s}^{-1}$. We thus argue that the $\sim 77 \mathrm{~km} \mathrm{~s}^{-1}$ gas is physically associated with SS 433/W50, which agrees well with the previous study by Lockman et al. (2007).

We note that the heliocentric systemic radial velocity of $56 \pm 2 \mathrm{~km} \mathrm{~s}^{-1}$ was suggested to be related to SS 433/W50 from deep optical observations toward the whole system (Boumis et al. 2007). The heliocentric radial velocity of $\sim 56 \mathrm{~km} \mathrm{~s}^{-1}$ can be transformed to a LSR velocity of $\sim 75 \mathrm{~km} \mathrm{~s}^{-1}$ (e.g., see Reid et al. 2009, 2014), which is in excellent agreement with our finding of $V_{\mathrm{LSR}}=77 \pm 5 \mathrm{~km} \mathrm{~s}^{-1}$ for the whole system on a large scale.

Accordingly, the LSR velocity of $77 \pm 5 \mathrm{~km} \mathrm{~s}^{-1}$, where the $\pm 5 \mathrm{~km} \mathrm{~s}^{-1}$ is the velocity error for possible peculiar motions, corresponds to a near kinematic distance of $4.9 \pm 0.4 \mathrm{kpc}$ (e.g., the A5 model in Reid et al. 2014). The far kinematic distance is excluded due to lacking $\mathrm{H}$ I absorption at the velocity near the tangent point (e.g., $V_{\mathrm{LSR}} \sim 85 \mathrm{~km} \mathrm{~s}^{-1}$ or $d_{\text {tangent }} \sim 6.4 \mathrm{kpc}$, see Figures 3 and 4 in Lockman et al. 2007).

We find that our new kinematic distance of $4.9 \pm 0.4 \mathrm{kpc}$ is somewhat smaller than the value of $5.5 \pm 0.2 \mathrm{kpc}$ from Lockman et al. (2007). The discrepancy of the two estimates comes from the different parameters of the Galactic rotation curve model. In the work of Lockman et al. (2007), they used a flat rotaion curve with $R_{0}=8.5 \mathrm{kpc}$ (distance of Sun from Galactic Center) and $V_{0}=220 \mathrm{~km} \mathrm{~s}^{-1}$ (rotation speed of Galaxy 
at $R_{0}$ ). Accordingly, the LSR velocity of $75 \pm 6 \mathrm{~km} \mathrm{~s}^{-1}$ from the atomic gas yielded the kinematic distance of $5.5 \pm 0.2 \mathrm{kpc}$. The velocity uncertainty of $\pm 6 \mathrm{~km} \mathrm{~s}^{-1}$ in their work is the typical random motion of cool H I clouds. In our case, we used the new Galactic rotation curve model, in which the values of $R_{0}=8.34 \mathrm{kpc}$ and $V_{0}=240 \mathrm{~km} \mathrm{~s}^{-1}$ are adopted (see Table 4 in Reid et al. 2014). Thus, the $77 \pm 5 \mathrm{~km} \mathrm{~s}^{-1}$ from the CO gas leads to a distance of $4.9 \pm 0.4 \mathrm{kpc}$. The velocity uncertainty of $\pm 5 \mathrm{~km} \mathrm{~s}^{-1}$, which is roughly comparable to the value of $\pm 10 \mathrm{~km} \mathrm{~s}^{-1}$ for the typical peculiar motions of the high mass star forming regions (Reid et al. 2014), is from the LSR velocity difference of the two MCs G39.315-1.155 and G40.331-4.302 (see Figures 6-9).

Finally, the distance to $\mathrm{SS} 433$ is estimated to be about $4.5-5.5 \mathrm{kpc}$ (depending on the different authors with somewhat different considerations, e.g., Hjellming \& Johnston 1981; Vermeulen et al. 1993; Stirling et al. 2002; Blundell \& Bowler 2004; Marshall et al. 2013; Panferov 2014) based on the kinematic model of the proper motions of the SS 433 jets. Our near kinematic distance of $4.9 \pm 0.4 \mathrm{kpc}$ is consistent well with the above estimates from the traditional kinematic model originally pioneered by Hjellming \& Johnston (1981).

We construct a cartoon to elucidate the association between the observed $\mathrm{CO}+\mathrm{H}$ I features and the SS 433/W50 system (Figure 13). The main features of the ISM surrounding SS 433/W50, such as the H I cavity near the Galactic plane, the H I wall toward the approaching gas from the SS 433 jet, and the corresponding kinematic characteristics of the gas and the jets, are all included in the map.

In Figure 13, the opening angle from $\mathrm{CO}$ data is about $\pm 10^{\circ}$, which is comparable to the results from the radio and X-ray studies of W50 at large distances (e.g., jet-ISM encounters at 35-80 arcmin from SS 433, Brinkmann et al. 1996; Dubner et al. 1998; Brinkmann et al. 2007), but much smaller than the $\pm 20^{\circ}$ of the current precession cone of the SS 433 jets at the inner region (e.g., $\lesssim 6$ arcsec from the compact source, Hjellming \& Johnston 1981; Stirling et al. 2002). The discrepancy of the two opening angles with respect to SS 433 is possibly due to the changing state of the jet precession with time (e.g., Kochanek \& Hawley 1990; Zavala et al. 2008; Goodall et al. 2011a) and/or some hydrodynamical recollimated mechanisms for a precessing jet (e.g., Eichler 1983; Peter \& Eichler 1993; Monceau-Baroux et al. 2015).

Whatever the exact scenarios, the violent jet-ISM interactions seem to be taking place around the precession axis of SS 433 with a small opening angle. The bulk kinetic energy and momentum of the high-velocity gas will produce the high-pressure environment, leading to the rapid $\mathrm{H}_{2}$ formation in such interaction regions (see Section 6). As a result, the new-formed molecular gas is likely distributed around an area near the outermost parts of the jet cones with a limited opening angle. On the other hand, the SS 433's jets can shock the ISM and produce the over-pressured environment with respect to the surrounding gas, which may inflate a bubble propagating away from the central source. The cavity-like structures of the atomic gas are probably such a case (e.g., the roughly "eight-shaped" morphology of the atomic gas in panel d of 
Figure 11). The size of the upper cavity-like feature of the atomic gas is much smaller than that of the lower one, which is also probably due to the relatively higher density environment close to the Galactic plane.

\section{THE FOSSIL RECORD OF JET-ISM INTERACTIONS}

SS 433 shows prominent jet activity, which deposits amounts of kinetic energy into its surrounding ISM from several tens astronomical units to dozens parsecs (e.g., see Figure 8 in Fabrika 2004). Now that the energetic jets of SS 433 may leave some mark on its ambient ISM, it is interesting to search for some corresponding counterparts of jets-ISM interactions. Several works has been done in earlier studies using radio (e.g., Hjellming \& Johnston 1981; Downes et al. 1986; Dubner et al. 1998; Blundell \& Bowler 2004; Lockman et al. 2007), X-ray (e.g., Watson et al. 1983; Brinkmann et al. 1996; Safi-Harb \& Ögelman 1997; Brinkmann et al. 2007), and optical (e.g., Zealey et al. 1980; Boumis et al. 2007) observations. In this Section, we propose that the energetic microquasar SS 433 probably has a more profound effect on its ambient ISM.

We turn our focus on the unusual MCs G39.315-1.155 and G40.331-4.302, which are outside the current radio boundaries of W50. Briefly, the intensity of ${ }^{12} \mathrm{CO}$ emission of the two MCs is about $1-3 \mathrm{~K}$ and shows non-Gaussian profiles, while the ${ }^{13} \mathrm{CO}$ emission of them is only marginally detected in some regions with relatively strong ${ }^{12} \mathrm{CO}$ emission (Figures 8 and 9 ). Both of the two MC concentrations exhibit fragmented structures with weak diffuse $\mathrm{CO}$ emission around the relatively strong $\mathrm{CO}$ peaks (see Figure 5). These fragmented clouds, which have somewhat different peak LSR velocities, display broad CO-line profiles (e.g., Figures 6-9).

We suggest that the two clouds are the fossil record of interactions between the SS 433 jets and the surrounding ISM. The evidence are summarized as follows:

(1) Spatial coincidence. The two MC concentrations are exactly aligned along the precession axis of SS 433 within a small opening angle of $\sim \pm 7^{\circ}$ (Section 3.4). Both of the MCs, which appear to be elongated from the northeast to the southwest, seem to form concave appearance or arc-shaped structure toward the direction of SS 433 (e.g., Figures 4, 5, and 12). The curvature of the two arc-like structures also points in the direction of SS 433. These features are similar to the laboratory experiments (e.g., see Figure 4 in Lebedev et al. 2002), magnetohydrodynamical simulations (e.g., Asahina et al. 2014), and other astrophysical systems with jet-ISM interactions (e.g., examples for the Herbig-Haro complex HH 1-2, Hester et al. 1998; the black hole Xray binary GRS 1915+105, Tetarenko et al. 2018; and the Seyfert 2 galaxy IC 5063, Oosterloo et al. 2000; Tadhunter et al. 2014; Morganti et al. 2015).

(2) Kinematic features. The kinematics of the two clouds is consistent well with the jet properties of SS 433. The receding jet of SS 433 may be responsible for the redshifted feature of G39.315-1.155 and the approaching jet is responsible for the blueshifted feature of G40.331-4.302 (Section 4). The CO emission of the MCs also 
exhibits broad line profiles and multi-peaks with slightly different LSR velocities (Figures 6-9). The angular distance of MC G39.315-1.155 from SS 433 is much nearer than MC G40.331-4.302, which agrees with the fact that MC G39.315-1.155 is becoming much more turbulent (Section 3.4) in the relatively high-density gas environment close to the Galactic plane. The high turbulence and multiple gas components in the clouds may originate from the shock process of SS 433.

The dynamical timescale of the jet process can be estimated as $\sim \frac{\text { length }}{\text { velocity }} \gtrsim 2 \times$ $10^{3}$ years when we consider the possible deceleration of the jets. Here, the angular distance of G40.331-4.302 from SS 433 is measured to be $\sim 2.15$ (or $\sim 180$ pc at a distance of $4.9 \mathrm{kpc}$ ) and the jet velocity is assumed to be at a constant of $\lesssim 0.26 \mathrm{c}$.

On the other hand, the powerful jets of SS 433 may accumulate considerable material at the end of the jet shock. For G40.331-4.302, the velocity difference along the LOS (or the radial velocity component) is about $7 \mathrm{~km} \mathrm{~s}^{-1}$ (Figures 7, 9, and 12). Assuming that the gas moves roughly along the precession axis of the SS 433 jets, the total velocity of the gas is $\frac{\Delta V_{\mathrm{LSR}}}{\cos (i)} \sim 40 \mathrm{~km} \mathrm{~s}^{-1}$, where $i$ represents the inclination angle of the shock to our LOS (e.g., $i \approx 80^{\circ}$, Margon 1984; Vermeulen et al. 1993; Eikenberry et al. 2001).

The atomic gas surrounding MC G40.331-4.302 displays multilayers with several $\mathrm{km} \mathrm{s}^{-1}$ velocity difference (e.g., the red, green, and blue emission in Figure 12). The separation of the atomic-gas layers is measured to be $\sim 4^{\prime}$ (or $5.7 \mathrm{pc}$ at a distance of $4.9 \mathrm{kpc}$ ), which is approximately half of the thickness of the whole gas slab (Section 3.4 ). Using the above velocity of the shocked molecular gas of $\sim 40 \mathrm{~km} \mathrm{~s}^{-1}$, we obtain the dynamical age of the moving gas of $\frac{3}{5} \times \frac{5.7 \mathrm{pc}}{40 \mathrm{~km} \mathrm{~s}^{-1}} \sim 0.8 \times 10^{5}$ years (e.g., $\Delta l_{\text {gas }} \propto t^{3 / 5}$, Kaiser \& Alexander 1997). The dynamical age of the approaching atomic gas is much larger than the timescale of the current jet process, supporting our hypothesis of previous dynamical interactions between the SS 433 jets and its surrounding ISM at $\sim 10^{5}$ years ago.

The kinetic energy of the disturbed gas around G40.331-4.302 is estimated to be $\sim 5 \times 10^{49} \mathrm{erg}$ (e.g., gas mass $\sim 3 \times 10^{3} M_{\odot}$ and velocity $\sim 40 \mathrm{~km} \mathrm{~s}^{-1}$ ). Adopting the kinetic luminosity of $\gtrsim 10^{39} \mathrm{erg} \mathrm{s}^{-1}$ for the jets (e.g., Fabrika 2004; Begelman et al. 2006), the kinetic energy of SS 433 are sufficient to power the disturbed gas on the timescale of $\lesssim 0.8 \times 10^{5}$ years for the energy transfer efficiency of $2 \%$. On consideration of possible intermittent jet activities of SS 433, higher values of the kinetic energy input and/or the energy transfer efficiency are possible.

Recently, van den Heuvel et al. (2017) proposed that the SS 433 system, which consists of a compact object of $4.3 \pm 0.8 M_{\odot}$ and a supergiant donor star of $12.3 \pm 3.3 M_{\odot}$ (Hillwig \& Gies 2008), can stably survive for the timescale of $10^{4}-10^{5}$ years. Because the massive donor has a radiative envelope, the system can avoid going into a commonenvelope (CE) phase and is able to gently spiral in with stable Roche lobe overflow (King \& Begelman 1999; King et al. 2000; van den Heuvel et al. 2017). The timescale of $\sim 10^{4}-10^{5}$ years is controlled by the thermal timescale of the envelope of the 12.3 
$M_{\odot}$ A-supergiant in the SS 433 system (van den Heuvel et al. 2017). We find that such the timescale is also consistent with the average time spend in the appearance of the binary systems as ultraluminous X-ray sources (ULXs) state of $\sim 10^{5}$ years (Pavlovskii et al. 2017).

For the moving atomic gas surrounding G40.331-4.302, the dynamical age of $0.8 \times 10^{5}$ years estimated above is comparable to the evolutionary timescale of the unusual Be/X-ray binary with stable Roche lobe overflow (e.g., for the case of the mass ratio <3.5, van den Heuvel et al. 2017), which is also consistent with the rapid $\mathrm{H}_{2}$ formation timescale of $\lesssim 0.1 \mathrm{Myr}$ for the gas far away from the Galactic plane at the high pressure (see Section 6).

The previous jet episodes (or possible intermittent jet activities, e.g. Goodall et al. 2011a,b), the winds from the system (e.g., Begelman et al. 1980; Konigl 1983; Fabrika 2004; Begelman et al. 2006; Panferov 2017), and the loop magnetic field on the large scale (Farnes et al. 2017) may play significant roles in the formation of the SS 433/W50's current configuration.

\section{FORMATION OF MOLECULAR GAS DUE TO JET-ISM INTERACTIONS?}

Figures 10-12 display that a wall structure is clearly seen toward the locus of the extension of the precession axis of the SS 433 jets, in which the column density of $\mathrm{HI}$ increased precipitously. Enhanced CO emissions are also found to be nicely associated with the wall structure seen in H I emission. MC G40.331-4.302 has the H I counterpart, which displays more extended arc-like structure nearly perpendicular to the precession axis of the SS 433 jets (Figure 12). The positional coincidence between G40.331-4.302 and the precession axis of SS 433, as well as their coincident kinematic features, strongly suggests the physical connection between them (see Sections 4 and 5).

We obtain that the column density, $N_{\mathrm{H}}=N(\mathrm{H} \mathrm{I})+2 \times N\left(\mathrm{H}_{2}\right)$, is about $2.1 \times 10^{21} \mathrm{~cm}^{-2}$ for the cloud G40.331-4.302, which corresponds to $A_{V} \approx 1.1 \mathrm{mag}$ assuming $N_{\mathrm{H}} \approx$ $1.9 \times 10^{21} \mathrm{~cm}^{-2} \mathrm{mag}^{-1} \times A_{V}$. At the column density, the molecules are shielded from UV radiation and self-shielding allows significant $\mathrm{H}_{2}$ molecules to exist. The total mass of the main concentration of the cloud is $\sim 3 \times 10^{3} M_{\odot}$ within a radius of $\sim 6{ }^{\prime} 6$ (Table 1). The molecular fraction $f_{\mathrm{H}_{2}}=2 N\left(\mathrm{H}_{2}\right) / N_{\mathrm{H}}$ is about 0.87 , suggesting that the dominant of the gas in the cloud is in molecular form.

The virial mass exceeds the total mass of the cloud by more than one order of magnitude, indicating that the cloud is in a gravitationally unbound state. The fragmented sub-clouds (e.g., with a size of $\sim 1$ pc) in MC G40.331-4.302 may dissipate on a timescale of several Myr (sound crossing time) without other supports. Alternatively, the MC may be confined by external pressure of $\gtrsim 4.4 \times 10^{4} \mathrm{~K} \mathrm{~cm}^{-3}$ (e.g., $\left.\rho(\mathrm{HI}) \sigma^{2}(\mathrm{HI})\right)$ when the magnetic pressure and the self-gravity are negligible.

We emphasize that G40.331-4.302 is the only detected MC within the range of $l=34^{\circ} 9-45^{\circ} .1,-5^{\circ} .1 \lesssim b \lesssim-3^{\circ} 0$, and $V_{\mathrm{LSR}} \gtrsim 70 \mathrm{~km} \mathrm{~s}^{-1}$. It indicates that such the 
$\mathrm{MC}$ is located about 370 pc below the Galactic plane, where a kinematic distance of $4.9 \mathrm{kpc}$ is adopted. However, the MCs within the solar circle are concentrated in the Galactic plane with an Gaussian FWHM of $\sim 100$ pc (e.g., see Section 4.5 and Figure 6 in Heyer \& Dame 2015). This suggests that G40.331-4.302 is an unusual MC at the locus far away from the Galactic plane. An interesting question is how to form the enormous molecular gas with the limited gas supply.

First of all, the formation timescale of the molecular gas, which was converted from the atomic gas, should be considered. The $\mathrm{H}_{2}$ formation timescale, $t_{\mathrm{H}_{2}} \simeq \frac{10^{9} \text { years }}{n\left(\mathrm{~cm}^{-3}\right)}$ (Hollenbach et al. 1971), is at least 10 Myr assuming the mean number density of $\sim 100 \mathrm{~cm}^{-3}\left(n_{\mathrm{H}}+2 \times n_{\mathrm{H}_{2}}\right.$, see Table 1$)$. However, the above estimation does not take account of dynamical processes, which are ubiquitous in the universe. The processes such as shocks, turbulence, and instabilities have a great impact on the effective $\mathrm{H}_{2}$ formation rate (e.g., Glover \& Mac Low 2007).

Here, we propose that the previous jets/outflows of SS 433 may play crucial roles in the process of molecular gas formation of G40.331-4.302. The $\mathrm{H}_{2}$ formation timescale can be simply written as, $t_{\mathrm{H}_{2}} \simeq 7 \times 10^{5} f_{\text {dust }}\left(\frac{2 \times 10^{5} \mathrm{~K} \mathrm{~cm}}{p_{\text {th }}}\right)^{0.95}$ years, connecting the $\mathrm{H}_{2}$ formation timescale with the thermal pressure (here $f_{\text {dust }}$ is the dust mass fraction remaining in the gas, Guillard et al. 2009). In our case, the ram pressure, which is defined as $p_{\text {ram }}=\rho v^{2}$, is probably dominant in the clouds of interest. For the ram pressure of $\sim 2 \times 10^{6} \mathrm{~K} \mathrm{~cm}^{-3}$ (e.g., $n \sim 7.3 \mathrm{~cm}^{-3}$ and $v \sim 40 \mathrm{~km} \mathrm{~s}^{-1}$ ), and $f_{\text {dust }}=1$, the $\mathrm{H}_{2}$ formation timescale is thus $\lesssim 0.1 \mathrm{Myr}$, indicating very rapid MC formation. Several numerical models were proposed that $\mathrm{H}_{2}$ formation occurs rapidly with dynamical processes considered (see, e.g., Koyama \& Inutsuka 2000; Bergin et al. 2004; Glover \& Mac Low 2007; Glover et al. 2010). The high external pressure due to the prevailing shock was also suggested to accelerate the transition from atomic gas to molecular gas in a short time (e.g., Röhser et al. 2014; Kaneko et al. 2017).

Furthermore, in the vicinity of G40.331-4.302 there may be considerable dark molecular gas (DMG) (e.g., Grenier et al. 2005; Abdo et al. 2010; Langer et al. 2010, 2014; Planck Collaboration et al. 2011), which is nearly invisible using CO rotational emission but considerable $\mathrm{H}_{2}$ molecules may exist in CO-dark or CO-faint regions. Simply speaking, the scale height $\left(l_{\text {height }}\right)$ of the gas layer probably follows the relationship of $l_{\text {height }}(\mathrm{H} \mathrm{I})>l_{\text {height }}(\mathrm{DMG})>l_{\text {height }}(\mathrm{CO})$ when we see the edge-on Milky Way. Once there exists sufficient $\mathrm{H}_{2}$ molecules, $\mathrm{CO}$ formation may occur rapidly in the turbulent DMG by considering the accumulation and compression of the material by ram pressure from the jet. The possible scenario can explain the unusual scale height of MC G40.331-4.302, which is nearly 370 pc below the Galactic plane. Observations such as $\mathrm{OH} 18 \mathrm{~cm}$ lines, $\mathrm{CH} 3.3 \mathrm{GHz}$ lines, and $158 \mu \mathrm{m}$ [CII] line toward the direction are advocated to investigate the nature of the gas for further study.

\section{SUMMARY}


In combination with $\mathrm{CO}$ data from the MWISP project and H I data from GBT $100 \mathrm{~m}$ and Arecibo $305 \mathrm{~m}$ telescopes, we investigate the large-scale ISM environment toward the SS 433/W50 system. Our main findings and comments are summarized as follows:

1. Two MC concentrations, MC G39.315-1.155 at $V_{\mathrm{LSR}} \sim 73-77 \mathrm{~km} \mathrm{~s}^{-1}$ and $\mathrm{MC}$ G40.331-4.302 at $V_{\mathrm{LSR}} \sim 77-84 \mathrm{~km} \mathrm{~s}^{-1}$, are found to be well aligned along the precession cone of SS 433 jets within a smaller opening angle of $\sim \pm 7^{\circ}$, indicating the possible connection between them. Further analysis show that kinematic features of the molecular gas are consistent with the characteristics of the SS 433 jets. That is, MC G39.315-1.155 toward the Galactic plane displays redshifted profiles for the receding gas, while MC G40.331-4.302 away from the Galactic plane does exhibit somewhat blueshifted features for the approaching material.

2. The two MCs have corresponding atomic features traced by H emission at $V_{\mathrm{LSR}} \sim 70-90 \mathrm{~km} \mathrm{~s}^{-1}$. For G39.315-1.155, the molecular gas is located at the top of the atomic gas cavity (see panel d in Figure 11), although the strong H I emission near the Galactic plane prevents us from analyzing its kinematic feature of the associated atomic gas. For G40.331-4.302, the H I emission also exhibits the blueshifted velocity structure, as well as that of the embedded molecular gas in the apex of the atomic gas wall. Additionally, the $\mathrm{H}$ I emission at such velocities actually displays the morphological resemblance with the radio nebula W50.

3. Based on the $\mathrm{CO}$ and $\mathrm{H}$ I data, the gas at $V_{\mathrm{LSR}}=77 \pm 5 \mathrm{~km} \mathrm{~s}^{-1}$ is suggested to be physically associated with the SS 433/W50 system. The LSR velocity of the associated gas from radio observations is consistent with those from optical observations. Accordingly, the near kinematic distance of the system is $4.9 \pm 0.4 \mathrm{kpc}$, which agrees well with the results from the kinematic model of the proper motions of the SS 433 jets within the error (e.g., see views in Panferov 2014). The far distance of the system can be excluded due to lacking the $\mathrm{H}$ I absorption near the tangent point, e.g., $V_{\mathrm{LSR}} \sim 85 \mathrm{~km} \mathrm{~s}^{-1}$.

4. The interesting MCs, which are exactly at the locus of the extension of the precession axis of the SS 433 jets, are outside the current boundaries of the extended nonthermal radio emission of W50. We argue that such gas is probably related to the very early process of the SS 433 jets and is the fossil record of jet-ISM interactions. Analyzing the moving atomic gas surrounding MC G40.331-4.302, we suggest that the dynamical process of the jet-ISM collisions probably began at $\sim 0.8 \times 10^{5}$ years ago.

5. Moreover, G40.331-4.302 is the only MC detected in CO emission at such a low latitude ( $\sim 370 \mathrm{pc}$ away from the Galactic plane). Connecting the blueshifted gas of G40.331-4.302 with the approaching jet of SS 433, we argue that the energetic jets of the microquasar have more profound effects on its ISM environment, leading to rapid MCs formation of $\lesssim 0.1 \mathrm{Myr}$ for the high ram pressure of $\sim 2 \times 10^{6} \mathrm{~K} \mathrm{~cm}^{-3}$. Such the rapid $\mathrm{H}_{2}$ formation timescale is also comparable to the dynamical age of the 
moving atomic gas around MC G40.331-4.302 and the evolutionary timescale of the unusual binary system (e.g., a low-mass black hole and a relatively moderate-mass donor star, van den Heuvel et al. 2017).

6. There may be considerable $\mathrm{H}_{2}$ gas with a little $\mathrm{CO}$ emission in the surrounding of the fragmented MC G40.331-4.302. We are advocating that further observations such as $\mathrm{OH} 18 \mathrm{~cm}$ lines, $\mathrm{CH} 3.3 \mathrm{GHz}$ lines, and $158 \mu \mathrm{m}$ [CII] line are helpful in investigating the nature of the $\mathrm{CO}$-faint $\mathrm{MC}$ and the nearby regions.

The authors acknowledge the staff members of the Qinghai Radio Observing Station at Delingha for their support of the observations. We would like to thank the anonymous referee for valuable comments and suggestions that helped to improve this paper. This work is supported by the National Key R\&D Program of China through grants 2017YFA0402701, 2017YFA0402702, 2017YFA0402600 and 2015CB857100. Y.S. thanks F. J. Lockman for providing the GBT HI data in our preliminary study. Y.C. thanks the National Natural Science Foundation of China for the support through grants 11773014, 11633007 and 11851305. Y.C. acknowledges support by the NSFC through grant 11473069. This publication utilizes data from Galactic ALFA HI (GALFA HI) survey data set obtained with the Arecibo L-band Feed Array (ALFA) on the Arecibo 305m telescope. Arecibo Observatory is part of the National Astronomy and Ionosphere Center, which is operated by Cornell Univer-

sity under Cooperative Agreement with the U.S. National Science Foundation. The GALFA HI surveys are funded by the NSF through grants to Columbia University, the University of Wisconsin, and the University of California.

Facility: PMO $13.7 \mathrm{~m}$

Software: GILDAS/CLASS (Pety 2005)

\section{REFERENCES}

Abdo, A. A., Ackermann, M., Ajello, M., et al. 2010, ApJ, 710, 133

Abolmasov, P., Maryeva, O., \& Burenkov, A. N. 2010, Astronomische Nachrichten, 331, 412

Acero, F., Ackermann, M., Ajello, M., et al. 2015, ApJS, 218, 23

Alves, M. I. R., Davies, R. D., Dickinson, C., et al. 2012, MNRAS, 422, 2429

Asahina, Y., Ogawa, T., Kawashima, T., et al. 2014, ApJ, 789, 79

Band, D. L. 1987, PASP, 99, 1269

Band, D. L., \& Gordon, M. A. 1989, ApJ, 338, 945
Begelman, M. C., Hatchett, S. P., McKee, C. F., Sarazin, C. L., \& Arons, J. 1980, ApJ, 238, 722

Begelman, M. C., King, A. R., \& Pringle, J. E. 2006, MNRAS, 370, 399

Bergin, E. A., Hartmann, L. W., Raymond, J. C., \& Ballesteros-Paredes, J. 2004, ApJ, 612, 921

Blundell, K. M., \& Bowler, M. G. 2004, ApJL, 616, L159

Bolatto, A. D., Wolfire, M., \& Leroy, A. K. 2013, ARA\&A, 51, 207

Boumis, P., Meaburn, J., Alikakos, J., et al. 2007, MNRAS, 381, 308 
Brinkmann, W., Aschenbach, B., \& Kawai, N. 1996, A\&A, 312, 306

Brinkmann, W., Pratt, G. W., Rohr, S., Kawai, N., \& Burwitz, V. 2007, A\&A, 463, 611

Broderick, J. W., Fender, R. P., Miller-Jones, J. C. A., et al. 2018, MNRAS, doi:10.1093/mnras/sty081

Chaty, S., Rodríguez, L. F., Mirabel, I. F., et al. 2001, A\&A, 366, 1035

Dame, T. M., Hartmann, D., \& Thaddeus, P. 2001, ApJ, 547, 792

Dickey, J. M., \& Lockman, F. J. 1990, ARA\&A, 28, 215

Downes, A. J. B., Pauls, T., \& Salter, C. J. 1986, MNRAS, 218, 393

Downes, A. J. B., Salter, C. J., \& Pauls, T. 1981, A\&A, 103, 277

Dubner, G. M., Holdaway, M., Goss, W. M., \& Mirabel, I. F. 1998, AJ, 116, 1842

Dubout-Crillon, R. 1976, A\&AS, 25, 25

Durouchoux, P., Sood, R., Oka, T., et al. 2000, Advances in Space Research, 25, 703

Eichler, D. 1983, ApJ, 272, 48

Eikenberry, S. S., Cameron, P. B., Fierce, B. W., et al. 2001, ApJ, 561, 1027

Elston, R., \& Baum, S. 1987, AJ, 94, 1633

Fabrika, S. 2004, Astrophysics and Space Physics Reviews, 12, 1

Farnes, J. S., Gaensler, B. M., Purcell, C., et al. 2017, MNRAS, 467, 4777

Gao, X. Y., Han, J. L., Reich, W., et al. 2011, A\&A, 529, A159

Geldzahler, B. J., Pauls, T., \& Salter, C. J. 1980, A\&A, 84, 237

Glover, S. C. O., Federrath, C., Mac Low, M.-M., \& Klessen, R. S. 2010, MNRAS, 404,2

Glover, S. C. O., \& Mac Low, M.-M. 2007, ApJ, 659, 1317

Goodall, P. T., Alouani-Bibi, F., \& Blundell, K. M. 2011a, MNRAS, 414, 2838

Goodall, P. T., Blundell, K. M., \& Bell Burnell, S. J. 2011b, MNRAS, 414, 2828

Green, D. A. 2017, VizieR Online Data Catalog, 7278
Green, G. M., Schlafly, E. F., Finkbeiner, D. P., et al. 2015, ApJ, 810, 25

Grenier, I. A., Casandjian, J.-M., \&

Terrier, R. 2005, Science, 307, 1292

Guillard, P., Boulanger, F., Pineau Des

Forêts, G., \& Appleton, P. N. 2009,

A\&A, 502, 515

Hester, J. J., Stapelfeldt, K. R., \&

Scowen, P. A. 1998, AJ, 116, 372

Heyer, M., \& Dame, T. M. 2015,

ARA\&A, 53, 583

Hillwig, T. C., \& Gies, D. R. 2008, ApJL, 676, L37

Hjellming, R. M., \& Johnston, K. J. 1981, ApJL, 246, L141

Hollenbach, D. J., Werner, M. W., \& Salpeter, E. E. 1971, ApJ, 163, 165

Huang, Y.-L., Dame, T. M., \& Thaddeus, P. 1983, ApJ, 272, 609

Kaiser, C. R., \& Alexander, P. 1997, MNRAS, 286, 215

Kaneko, H., Kuno, N., Iono, D., et al. 2017, PASJ, 69, 66

King, A. R., \& Begelman, M. C. 1999, ApJL, 519, L169

King, A. R., Taam, R. E., \& Begelman, M. C. 2000, ApJL, 530, L25

Kochanek, C. S., \& Hawley, J. F. 1990, ApJ, 350, 561

Konigl, A. 1983, MNRAS, 205, 471

Koyama, H., \& Inutsuka, S.-I. 2000, ApJ, 532, 980

Langer, W. D., Velusamy, T., Pineda, J. L., et al. 2010, A\&A, 521, L17

Langer, W. D., Velusamy, T., Pineda, J. L., Willacy, K., \& Goldsmith, P. F. 2014, A\&A, 561, A122

Lebedev, S. V., Chittenden, J. P., Beg, F. N., et al. 2002, ApJ, 564, 113

Lockman, F. J., Blundell, K. M., \& Goss, W. M. 2007, MNRAS, 381, 881

Margon, B. 1984, ARA\&A, 22, 507

Marshall, H. L., Canizares, C. R., Hillwig, T., et al. 2013, ApJ, 775, 75

Mazeh, T., Aguilar, L. A., Treffers, R. R., Konigl, A., \& Sparke, L. S. 1983, ApJ, 265, 235

Mirabel, I. F., Claret, A., Cesarsky, C. J., Boulade, O., \& Cesarsky, D. A. 1996, A\&A, 315, L113 
Moldowan, A., Safi-Harb, S., Fuchs, Y., \& Dubner, G. 2005, Advances in Space Research, 35, 1062

Monceau-Baroux, R., Porth, O., Meliani, Z., \& Keppens, R. 2015, A\&A, 574, A143

Morganti, R., Oosterloo, T., Oonk, J. B. R., Frieswijk, W., \& Tadhunter, C. $2015, \mathrm{~A} \& \mathrm{~A}, 580, \mathrm{~A} 1$

Oosterloo, T. A., Morganti, R., Tzioumis, A., et al. 2000, AJ, 119, 2085

Paladini, R., Burigana, C., Davies, R. D., et al. 2003, A\&A, 397, 213

Panferov, A. 2014, A\&A, 562, A130

Panferov, A. A. 2017, A\&A, 599, A77

Pavlovskii, K., Ivanova, N., Belczynski, K., \& Van, K. X. 2017, MNRAS, 465, 2092

Peek, J. E. G., Heiles, C., Douglas, K. A., et al. 2011, ApJS, 194, 20

Peter, W., \& Eichler, D. 1993, ApJ, 417, 170

Pety, J. 2005, in SF2A-2005: Semaine de l'Astrophysique Francaise, ed. F. Casoli, T. Contini, J. M. Hameury, \& L. Pagani, 721

Planck Collaboration, Ade, P. A. R., Aghanim, N., et al. 2011, A\&A, 536, A19

Reich, W., Fuerst, E., Reich, P., \& Reif, K. 1990, A\&AS, 85, 633

Reid, M. J., Menten, K. M., Zheng, X. W., et al. 2009, ApJ, 700, 137

Reid, M. J., Menten, K. M., Brunthaler, A., et al. 2014, ApJ, 783, 130

Röhser, T., Kerp, J., Winkel, B., Boulanger, F., \& Lagache, G. 2014, A\&A, 564, A71

Safi-Harb, S., \& Ögelman, H. 1997, ApJ, 483, 868

Safi-Harb, S., \& Petre, R. 1999, ApJ, 512, 784

Shan, W. L., Yang, J., Shi, S. C., et al. 2012, IEEE Transactions on Terahertz Science and Technology, 2, 593
Stirling, A. M., Jowett, F. H., Spencer, R. E., et al. 2002, MNRAS, 337, 657

Su, Y., Fang, M., Yang, J., Zhou, P., \& Chen, Y. 2014a, ApJ, 788, 122

Su, Y., Sun, Y., Li, C., et al. 2016, ApJ, 828, 59

Su, Y., Yang, J., Zhou, X., Zhou, P., \& Chen, Y. 2014b, ApJ, 796, 122

Su, Y., Zhou, X., Yang, J., et al. 2017a, ApJ, 845, 48

—. 2017b, ApJ, 836, 211

Sun, Y., Su, Y., Zhang, S.-B., et al. 2017, ApJS, 230, 17

Tadhunter, C., Morganti, R., Rose, M., Oonk, J. B. R., \& Oosterloo, T. 2014, Nature, 511, 440

Tetarenko, A. J., Freeman, P., Rosolowsky, E. W., Miller-Jones, J. C. A., \& Sivakoff, G. R. 2018, MNRAS, 475, 448

van den Bergh, S. 1980, ApJL, 236, L23

van den Heuvel, E. P. J., Portegies Zwart, S. F., \& de Mink, S. E. 2017, MNRAS, 471,4256

Vermeulen, R. C., Schilizzi, R. T., Spencer, R. E., Romney, J. D., \& Fejes, I. 1993, A\&A, 270, 177

Vollmer, B., Gassmann, B., Derrière, S., et al. 2010, A\&A, 511, A53

Wang, Z.-R., McCray, R., Chen, Y., \& Qu, Q.-Y. 1990, A\&A, 240, 98

Watson, M. G., Willingale, R., Grindlay, J. E., \& Seward, F. D. 1983, ApJ, 273, 688

Yamamoto, H., Ito, S., Ishigami, S., et al. 2008, PASJ, 60, 715

Yamauchi, S., Kawai, N., \& Aoki, T. 1994, PASJ, 46, L109

Zavala, J., Velázquez, P. F., Cerqueira, A. H., \& Dubner, G. M. 2008, MNRAS, 387,839

Zealey, W. J., Dopita, M. A., \& Malin, D. F. 1980, MNRAS, 192, 731 
Table 1. Properties of Gas toward G39.315-1.155 and G40.331-4.302

\begin{tabular}{|c|c|c|c|c|c|c|c|}
\hline Name & Tracer & $\begin{array}{c}\text { Area }^{\mathrm{a}} \\
\left(\operatorname{arcmin}^{2}\right)\end{array}$ & $\begin{array}{l}\text { Radius }^{b} \\
\text { (arcmin) }\end{array}$ & $\begin{array}{c}I_{\text {mean }}{ }^{\mathrm{c}} \\
\left(\mathrm{K} \mathrm{km} \mathrm{s}^{-1}\right)\end{array}$ & $\begin{array}{l}\text { Column Density } \\
\left.\qquad \times 10^{20} \mathrm{~cm}^{-2}\right)\end{array}$ & $\begin{array}{c}\text { Mass }^{\mathrm{e}} \\
\left(\times 10^{3} M_{\odot}\right)\end{array}$ & $\begin{array}{l}\text { Volume Density } \mathrm{e}, \mathrm{f} \\
\qquad\left(\mathrm{cm}^{-3}\right)\end{array}$ \\
\hline G39.315-1.155 & ${ }^{12} \mathrm{CO}$ & 136.5 & 6.6 & 8.8 & $N\left(\mathrm{H}_{2}\right)=17.6$ & $M\left(\mathrm{H}_{2}\right)=10.7 d_{4.9}^{2}$ & $n\left(\mathrm{H}_{2}\right)=45.5 d_{4.9}^{-1}$ \\
\hline \multirow[t]{2}{*}{ G40.331-4.302 } & H I & 135.0 & 6.6 & 154.2 & $N(\mathrm{H})=2.8$ & $M(\mathrm{H})=0.8 d_{4.9}^{2}$ & $n(\mathrm{H})=7.3 d_{4.9}^{-1}$ \\
\hline & ${ }^{12} \mathrm{CO}$ & 53.3 & 4.1 & 4.6 & $N\left(\mathrm{H}_{2}\right)=9.2$ & $M\left(\mathrm{H}_{2}\right)=2.2 d_{4.9}^{2}$ & $n\left(\mathrm{H}_{2}\right)=38.2 d_{4.9}^{-1}$ \\
\hline
\end{tabular}

$a_{\text {The }}$ area of the emission with $I(\mathrm{CO})>5 \quad \mathrm{~K} \mathrm{~km} \mathrm{~s}^{-1}$ for $\mathrm{G} 39.315-1.155$ and $I(\mathrm{H} \mathrm{I})>130 \mathrm{~K} \mathrm{~km} \mathrm{~s}^{-1}$ and $I(\mathrm{CO})>2.5 \mathrm{~K} \mathrm{~km} \mathrm{~s}^{-1}$ for $\mathrm{G} 40.331-4.302$.

$\left.b_{\text {The effective radius is calculated from (Area/3.14) }}\right)^{0.5}$.

${ }^{c}$ The mean intensity of the $\mathrm{HI}\left(70-90 \mathrm{~km} \mathrm{~s}^{-1}\right)$ and CO $\left(73-88 \mathrm{~km} \mathrm{~s}^{-1}\right)$ emission.

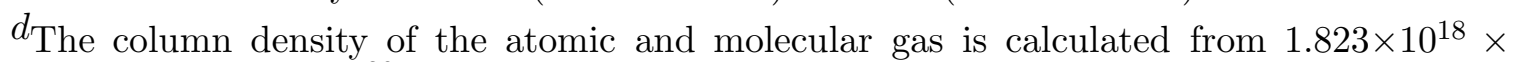
$I_{\text {mean }}(\mathrm{HI})$ and $2 \times 10^{20} \times I_{\text {mean }}(\mathrm{CO})$, respectively. See text.

${ }^{e}$ Parameter $d_{4.9}$ is the distance to the cloud in units of $4.9 \mathrm{kpc}$ (see Section 4).

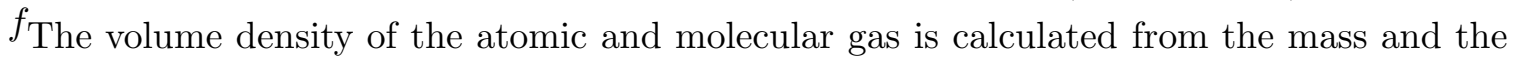
effective radius of the emission. 


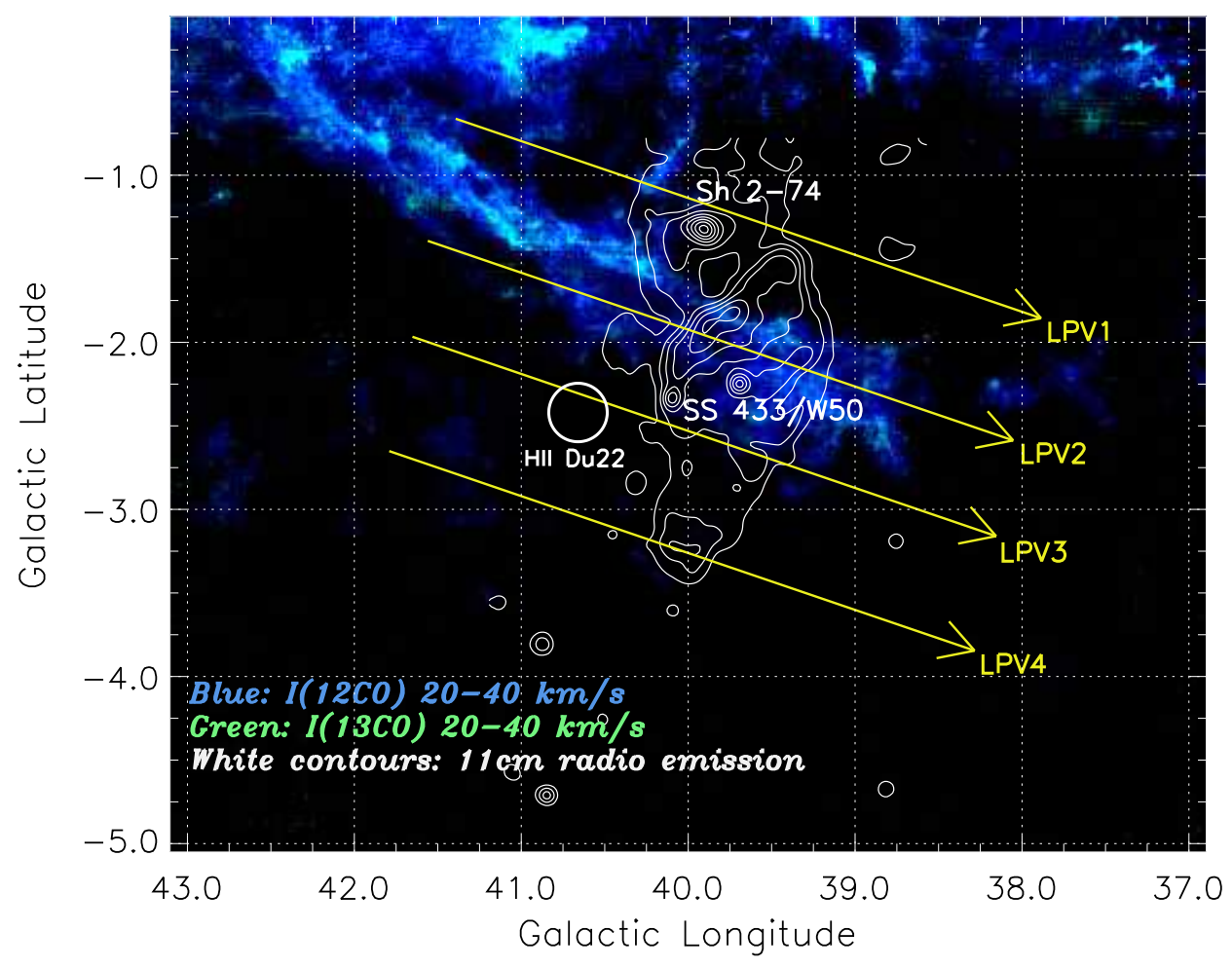

Figure 1. The MWISP ${ }^{12} \mathrm{CO}(J=1-0$, blue $)$ and ${ }^{13} \mathrm{CO}(J=1-0$, green $)$ intensity map toward SS $433 / \mathrm{W} 50$ in the $20-40 \mathrm{~km} \mathrm{~s}^{-1}$ interval, overlaid with radio continuum contours from the Effelsberg $11 \mathrm{~cm}$ survey (Reich et al. 1990). The nearby sources of H II regions Sh 2-74 and Du 22 are also labeled. The four yellow arrows indicate the PV slices shown in Figure 2. 

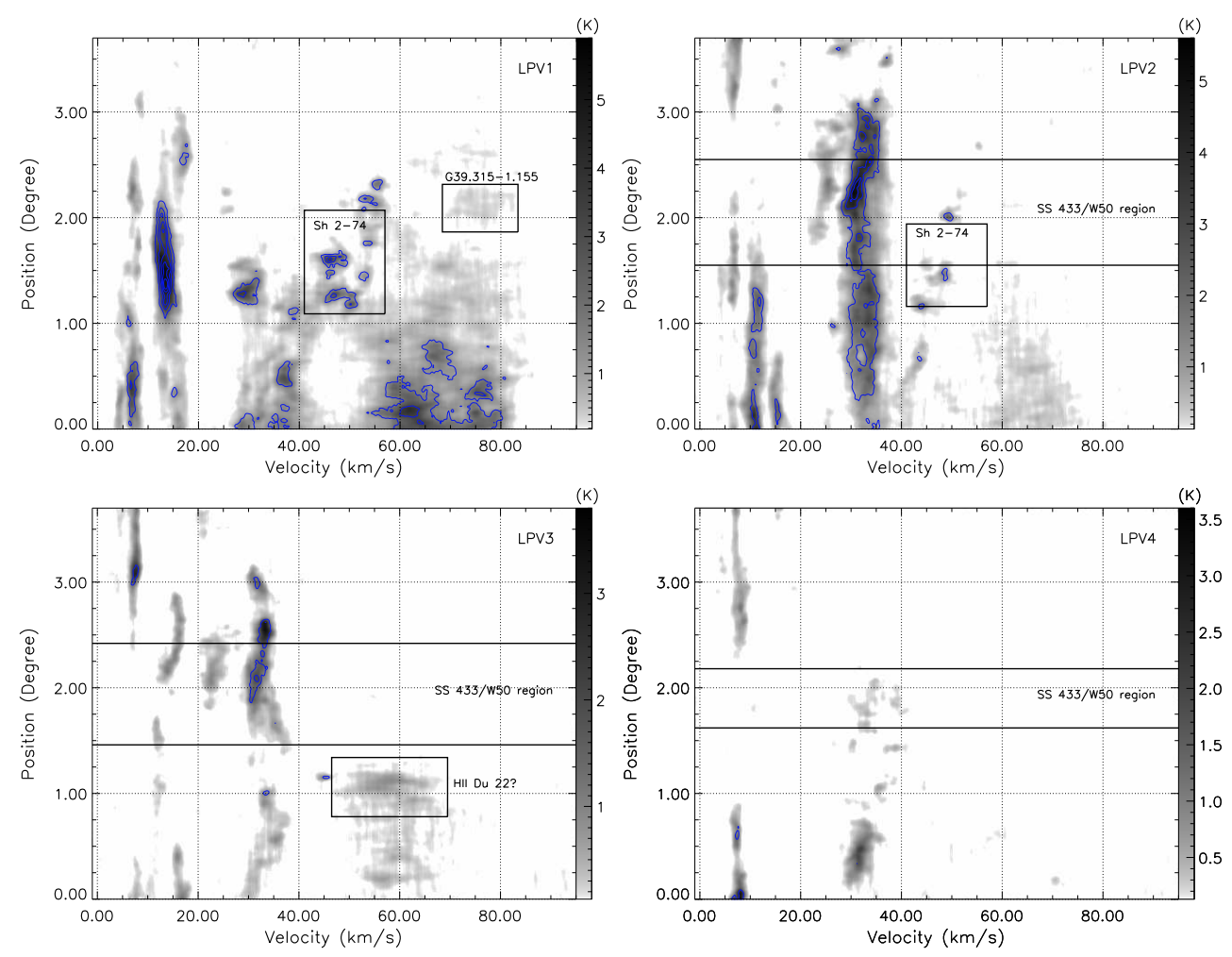

Figure 2. PV diagrams of ${ }^{12} \mathrm{CO}(J=1-0)$ emission along LPV1, LPV2, LPV3, and LPV4, overlaid with the blue contours of ${ }^{13} \mathrm{CO}$ emission. The overlaid contour levels start from $0.2 \mathrm{~K}$ and increase by a step of $0.4 \mathrm{~K}$. The PV slices have a length of $3.7((l=41.393$, $b=-0.662)$ to $(l=37.886, b=-1.856)$ for LPV1, $(l=41.560, b=-1.393)$ to $\left(l=38^{\circ} .053\right.$, $b=-2.587)$ for LPV2, $(l=41.652, b=-1.967)$ to $(l=38.155, b=-3.160)$ for LPV3, and $(l=41.791, b=-2.651)$ to $(l=38.284, b=-3.845)$ for LPV4 $)$ and a width of 0.508 . The horizontal lines show the radio regions of W50 nebula. 


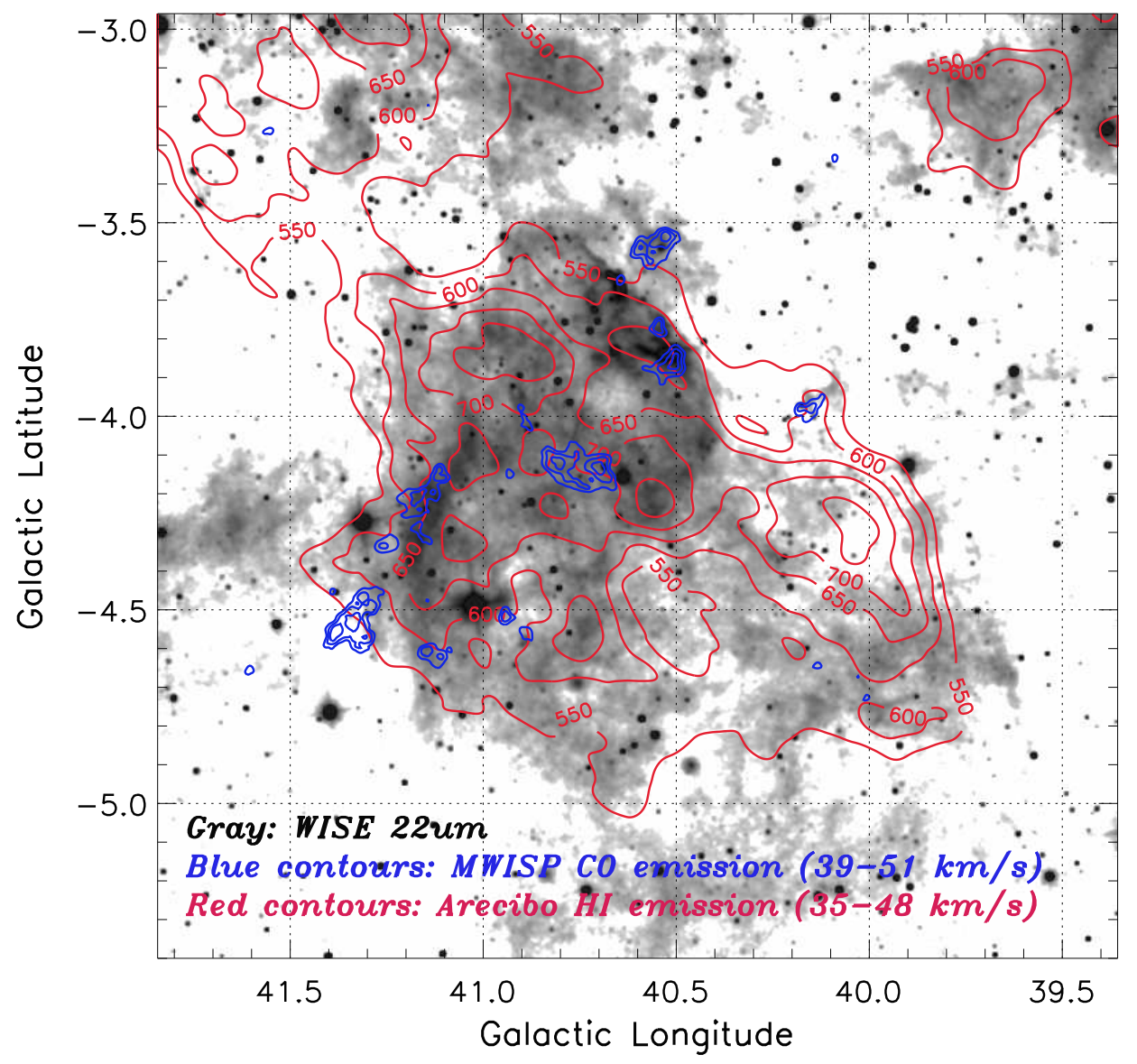

Figure 3. WISE $22 \mu \mathrm{m}$ emission toward $\left(l=40^{\circ} 4, b=-4.3\right)$, overlaid with molecular gas emission (blue contours with 3,6 , and $9 \mathrm{~K} \mathrm{~km} \mathrm{~s}^{-1}$ for the MWISP ${ }^{12} \mathrm{CO}$ in the interval of $39-51 \mathrm{~km} \mathrm{~s}^{-1}$ ) and atomic gas emission (red contours with 550, 600, 650, 700, and $750 \mathrm{~K} \mathrm{~km} \mathrm{~s}^{-1}$ for the GALFA H I in the interval of $35-48 \mathrm{~km} \mathrm{~s}^{-1}$ ). 


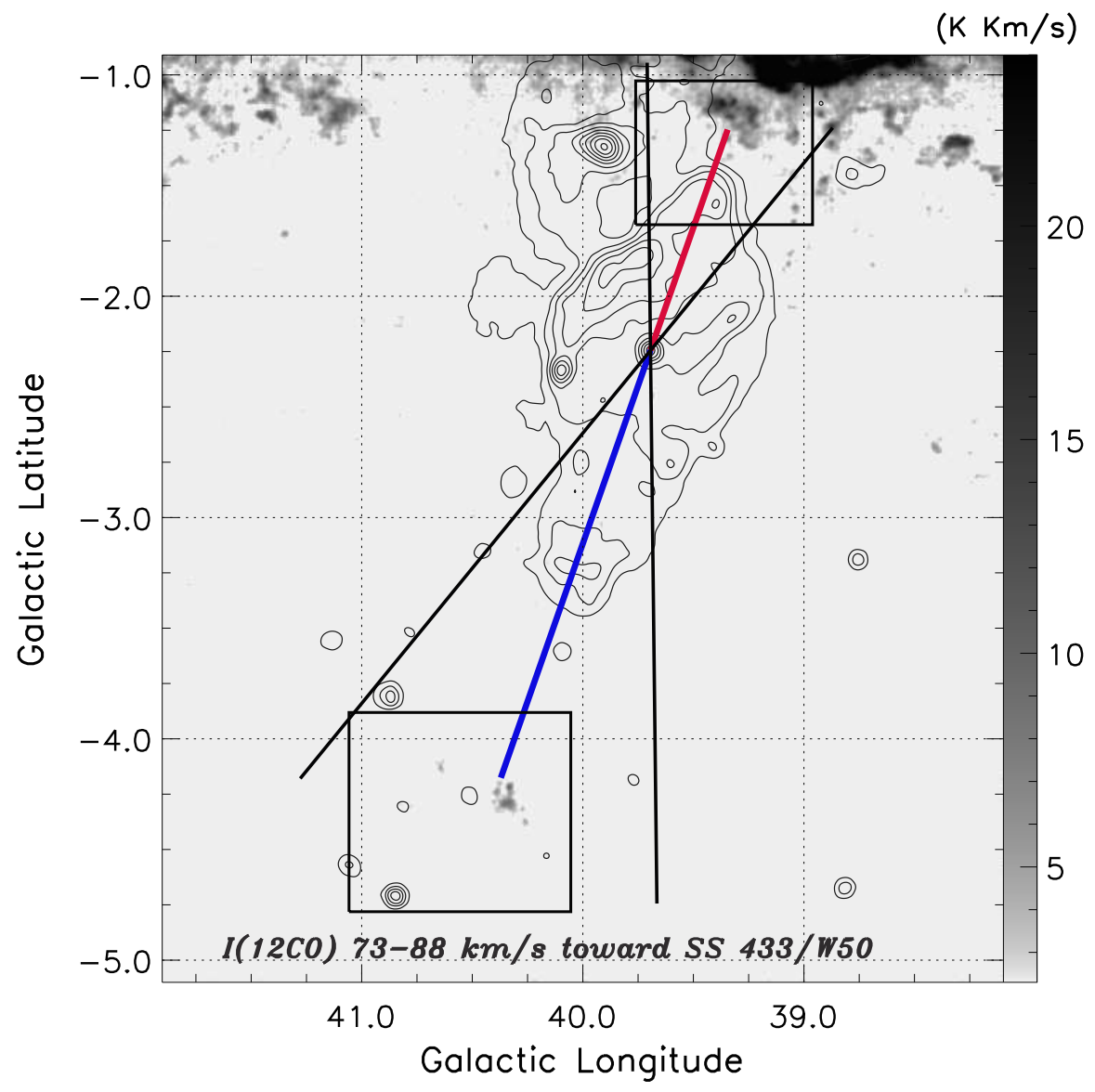

Figure 4. Integrated ${ }^{12} \mathrm{CO}(J=1-0)$ emission toward SS 433/W50 in the interval of 73$88 \mathrm{~km} \mathrm{~s}^{-1}$, overlaid with the same radio contours as in Figure 1. The thick red and blue line indicates the precession axis of the SS 433 jets. (see e.g., Hjellming \& Johnston 1981; Stirling et al. 2002). The black lines indicate the cone-opening angle of $\pm 20^{\circ}$ around the precession axis. The black boxes indicate the two regions shown in Figure 5. 

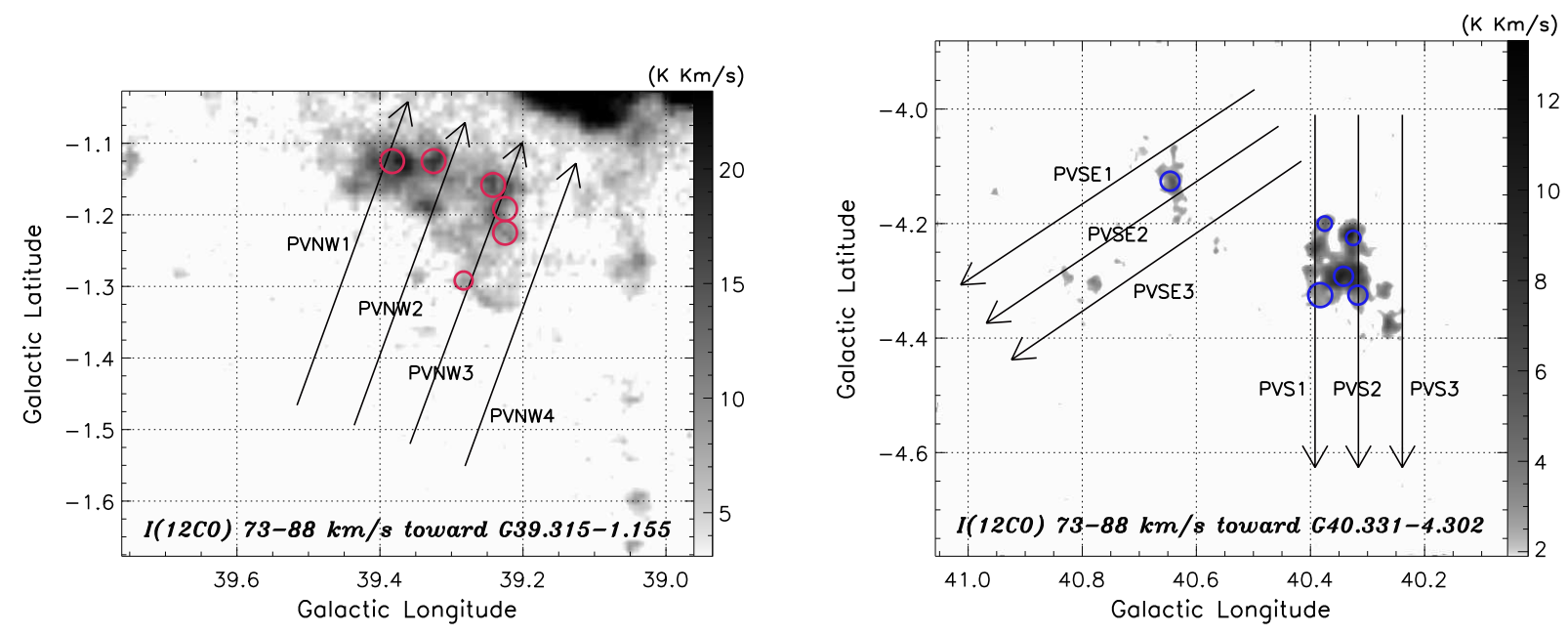

Figure 5. Left panel: ${ }^{12} \mathrm{CO}(J=1-0)$ emission toward the northwestern region (MC G39.315-1.155) of SS 433/W50. The four arrows indicate the PV slices shown in Figure 6. The six red circles indicate positions of the typical spectra shown in Figure 8. Right panel: ${ }^{12} \mathrm{CO}(J=1-0)$ emission toward the southeastern region (MC G40.331-4.302) of SS 433/W50. The six arrows indicate the PV slices shown in Figure 7. The six blue circles indicate positions of the typical spectra shown in Figure 9. 

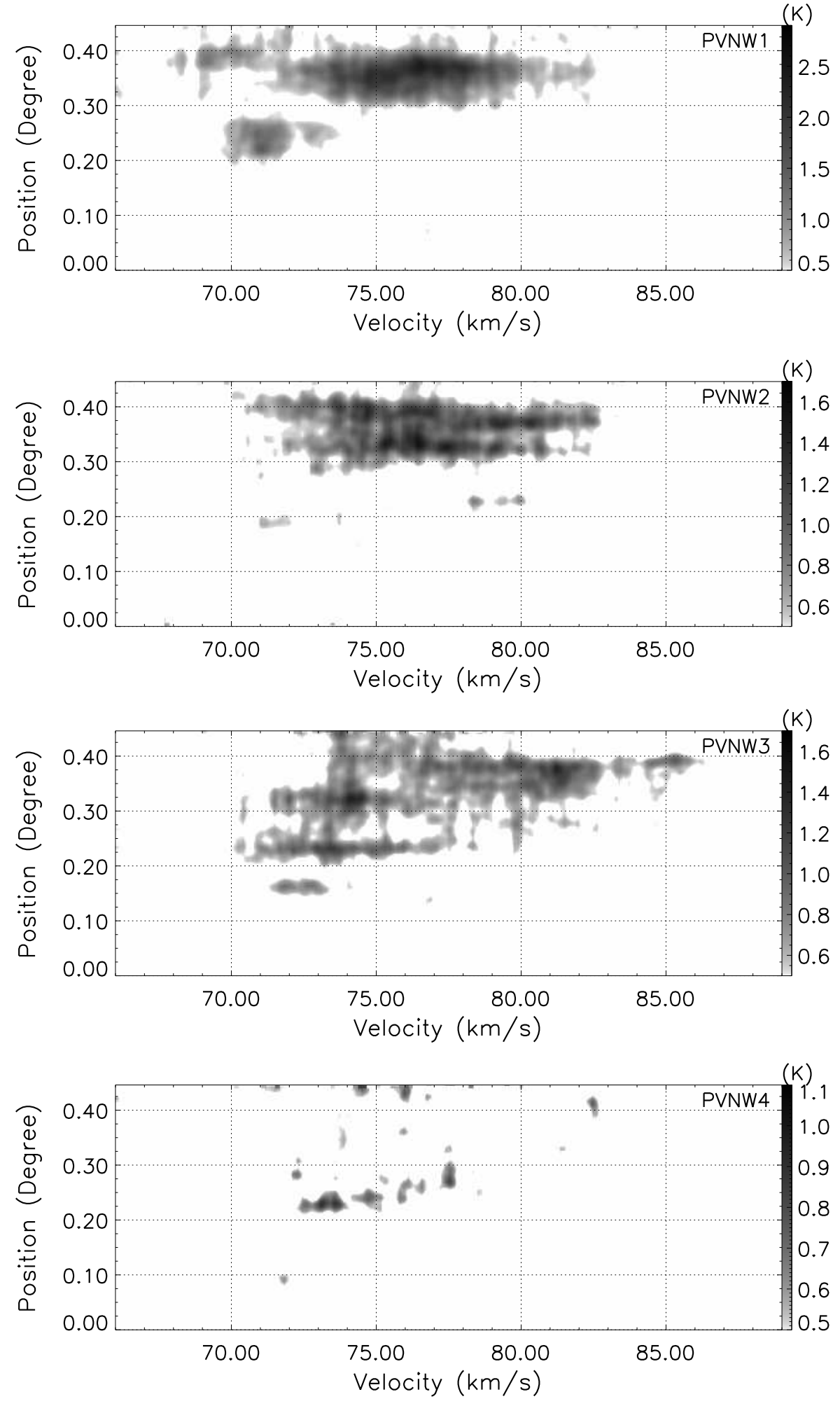

Figure 6. PV diagrams of ${ }^{12} \mathrm{CO}(J=1-0)$ emission along PVNW1, PVNW2, PVNW3, and PVNW4 for MC G39.315-1.155. The PV slices have a length of 26!8 and a width of 4.5 . 

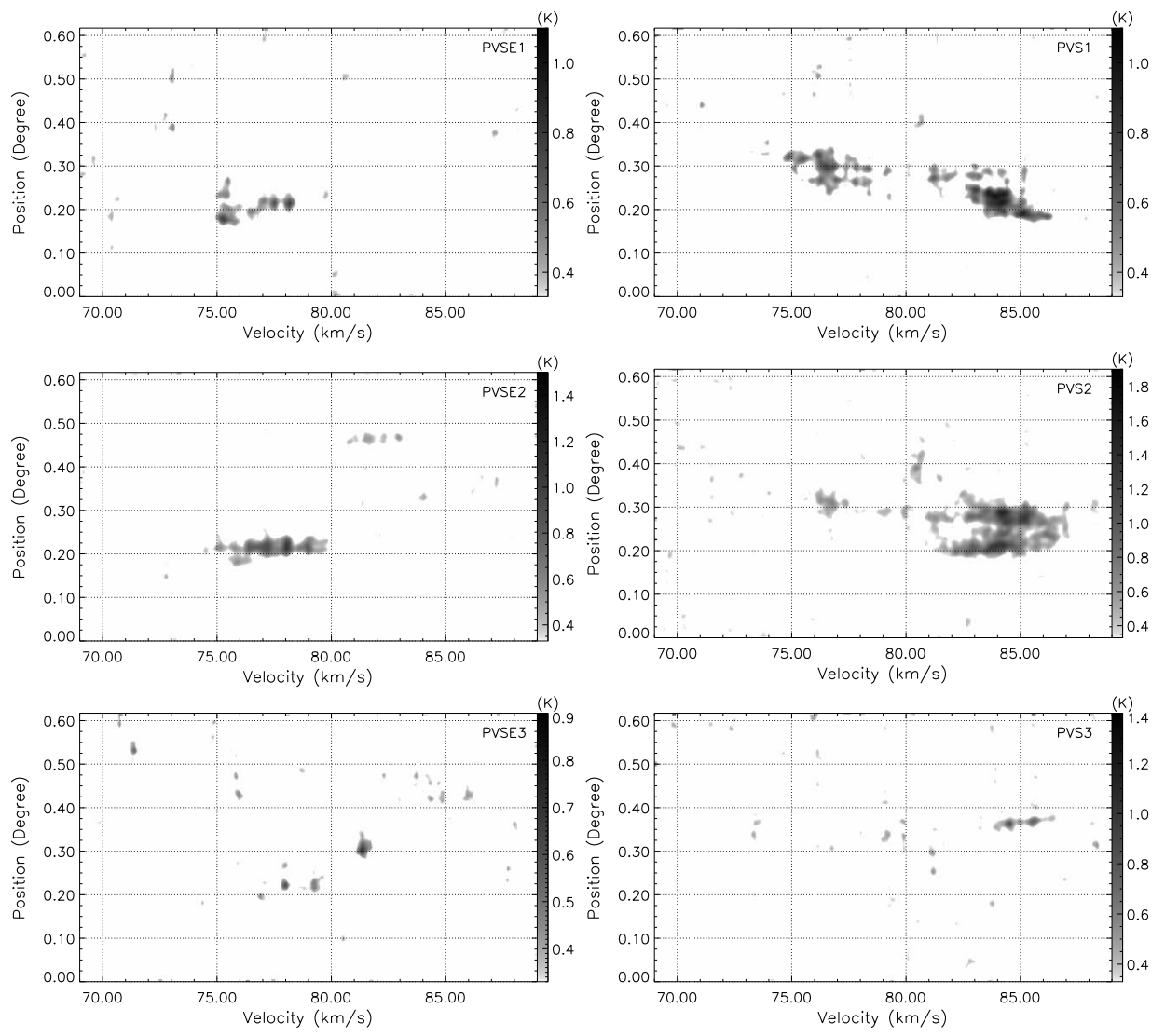

Figure 7. PV diagrams of ${ }^{12} \mathrm{CO}(J=1-0)$ emission along PVSE1, PVSE2, PVSE3, PVS1, PVS2, and PVS3 for MC G40.331-4.302. The PV slices have a length of 37!.0 and a width of 4.5 . 

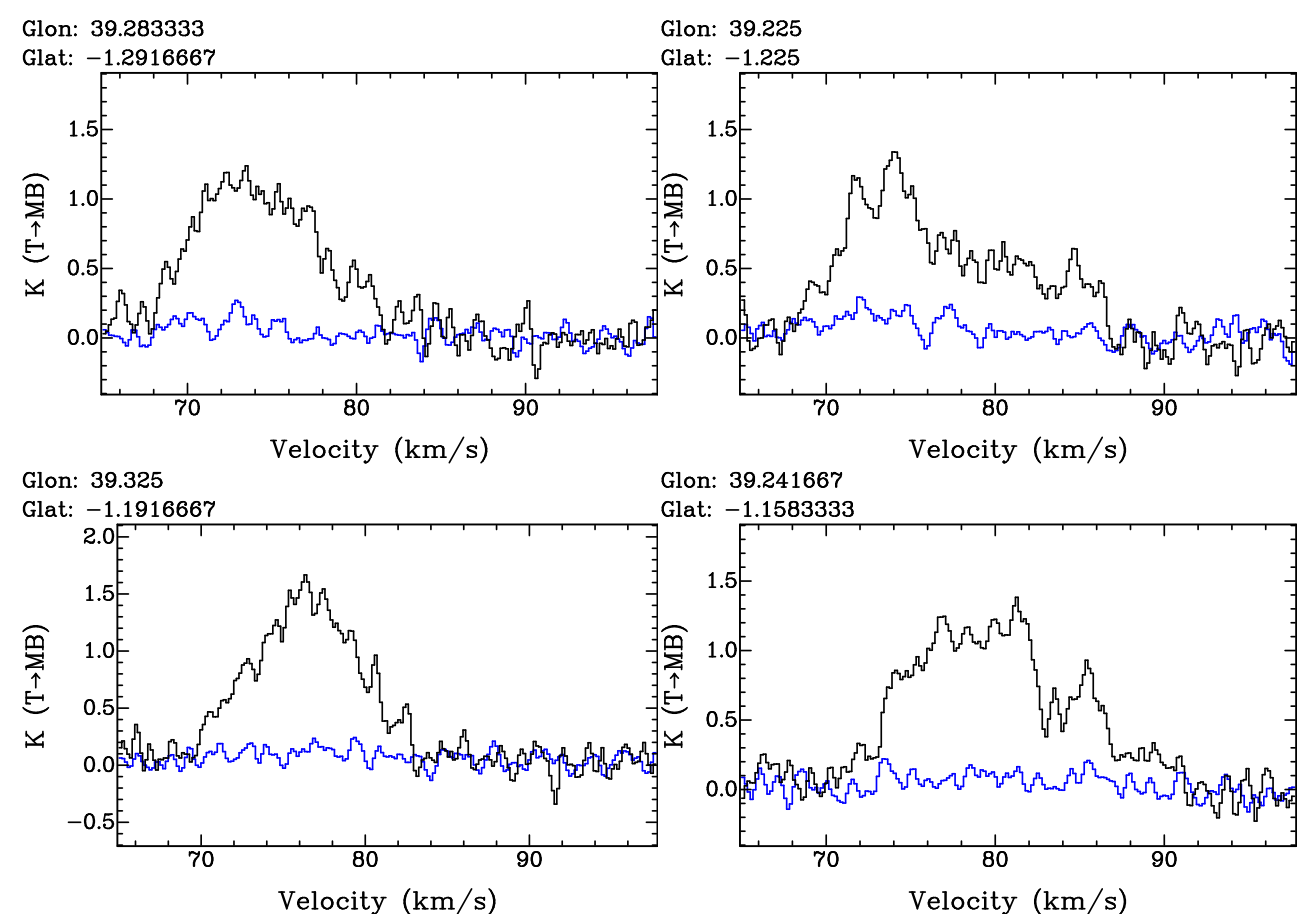

Glon: 39.241667
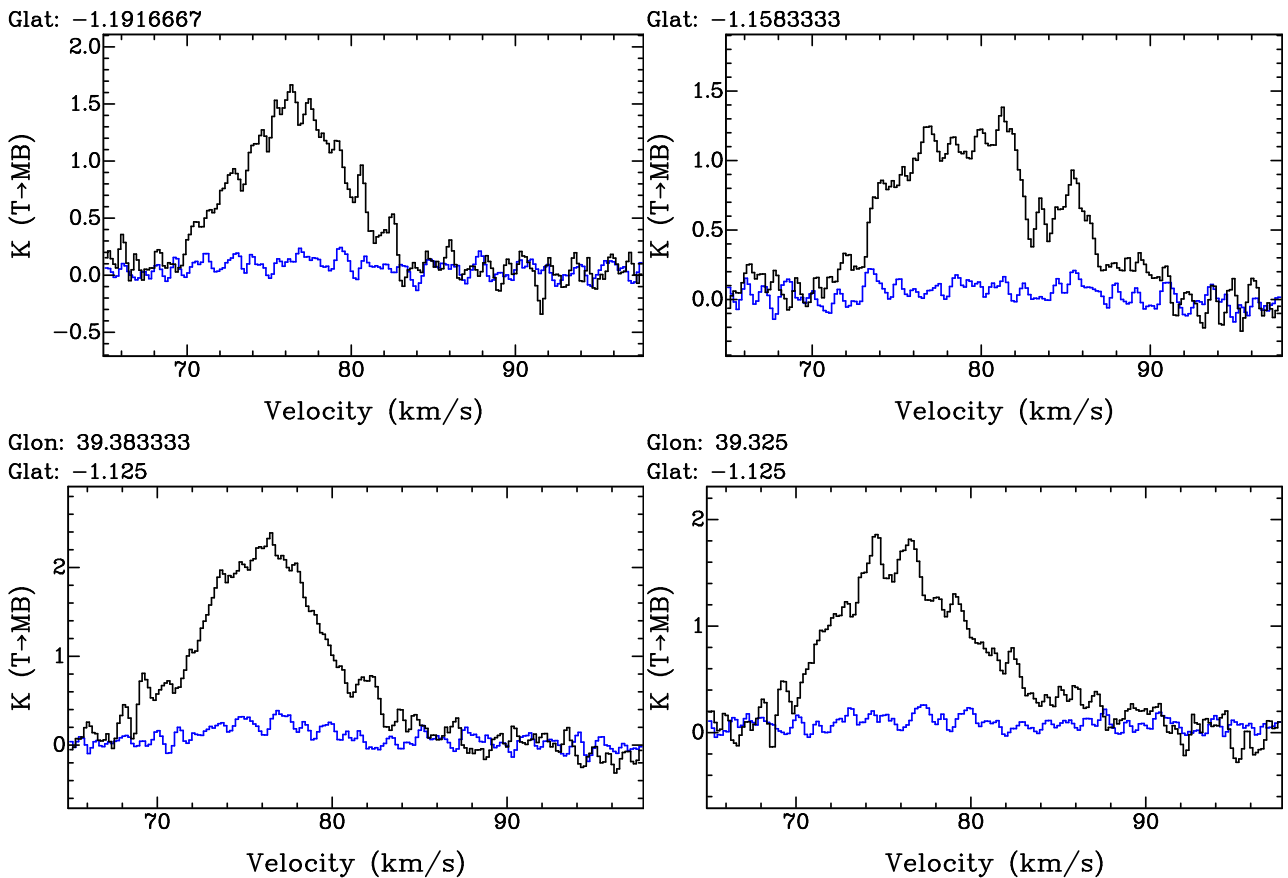

Glon: 39.325

Glat: -1.125

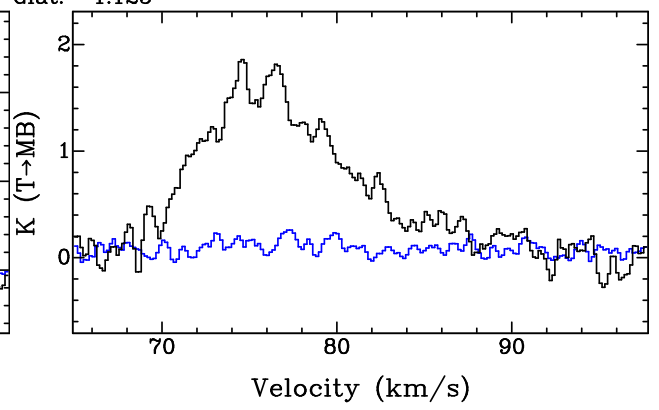

Figure 8. Typical ${ }^{12} \mathrm{CO}(J=1-0$; black $)$ and ${ }^{13} \mathrm{CO}(J=1-0$; blue $)$ spectra toward MC G39.315-1.155. 

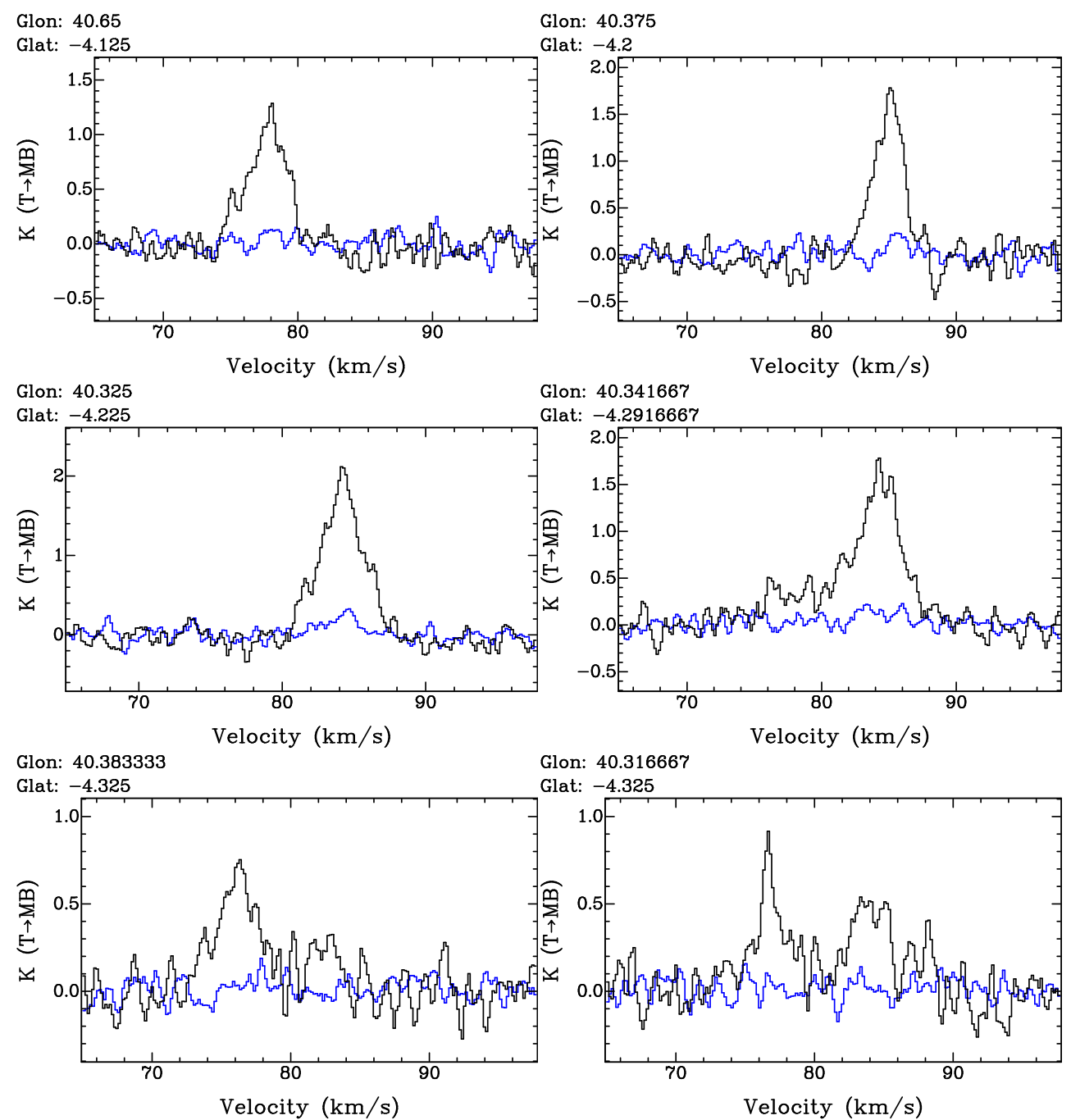

Glon: 40.316667 Glat: -4.325

Figure 9. Typical ${ }^{12} \mathrm{CO}(J=1-0$; black $)$ and ${ }^{13} \mathrm{CO}(J=1-0$; blue $)$ spectra toward MC G40.331-4.302. 

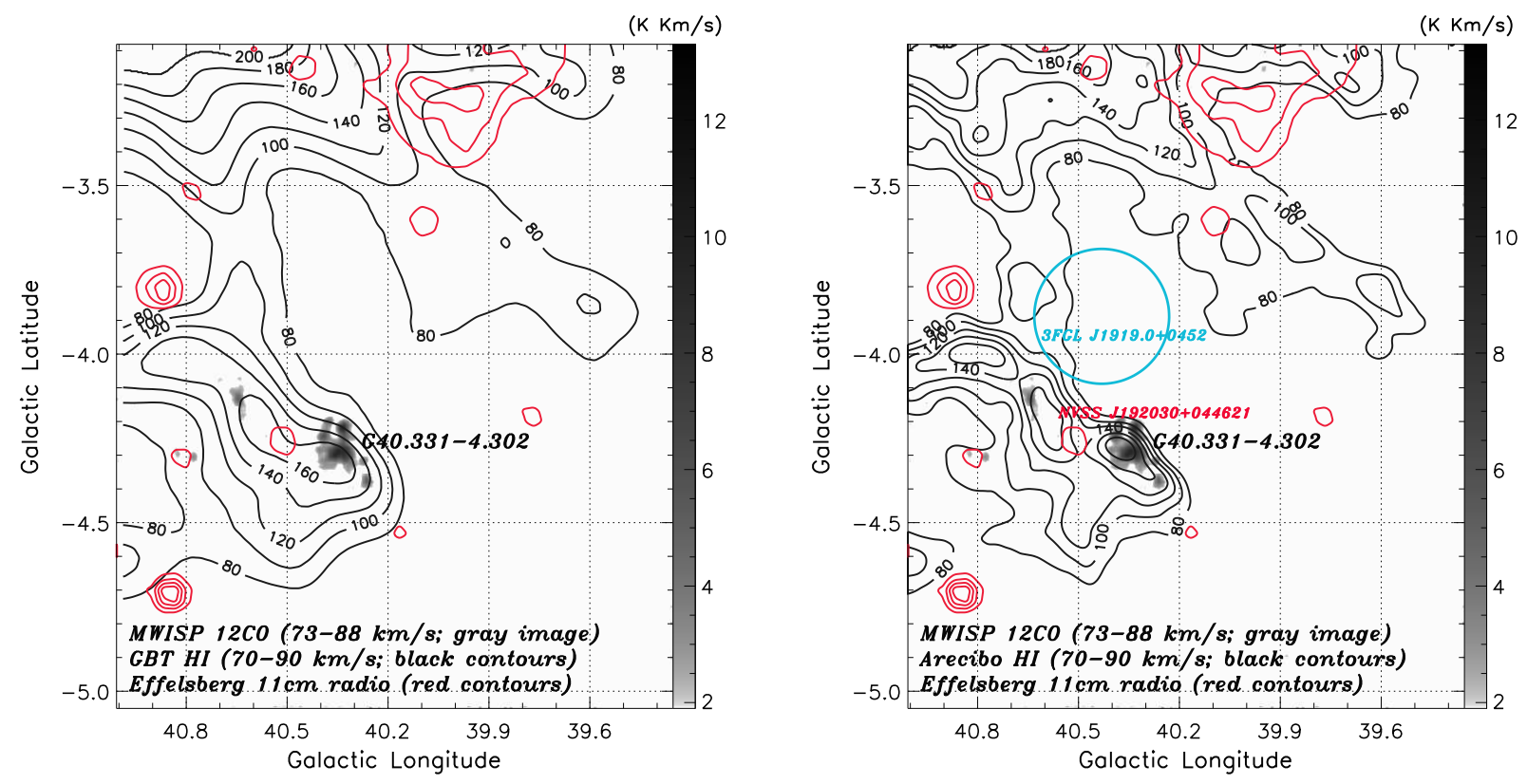

Figure 10. Left panel: Integrated ${ }^{12} \mathrm{CO}(J=1-0)$ emission in the interval of $73-88 \mathrm{~km} \mathrm{~s}^{-1}$ toward MC G40.331-4.302, overlaid with the same red radio contours as in Figure 1. The black contours show the GBT H I emission integrated in the interval of $70-90 \mathrm{~km} \mathrm{~s}^{-1}$. Right panel: Same as the left panel but overlaid with the H I contours from the Arecibo telescope. The cyan circle indicates the Fermi source of 3FGL J1919.0+0452 (Acero et al. 2015). The radio source of NVSS J192030+044621 (Vollmer et al. 2010) is also labeled. 


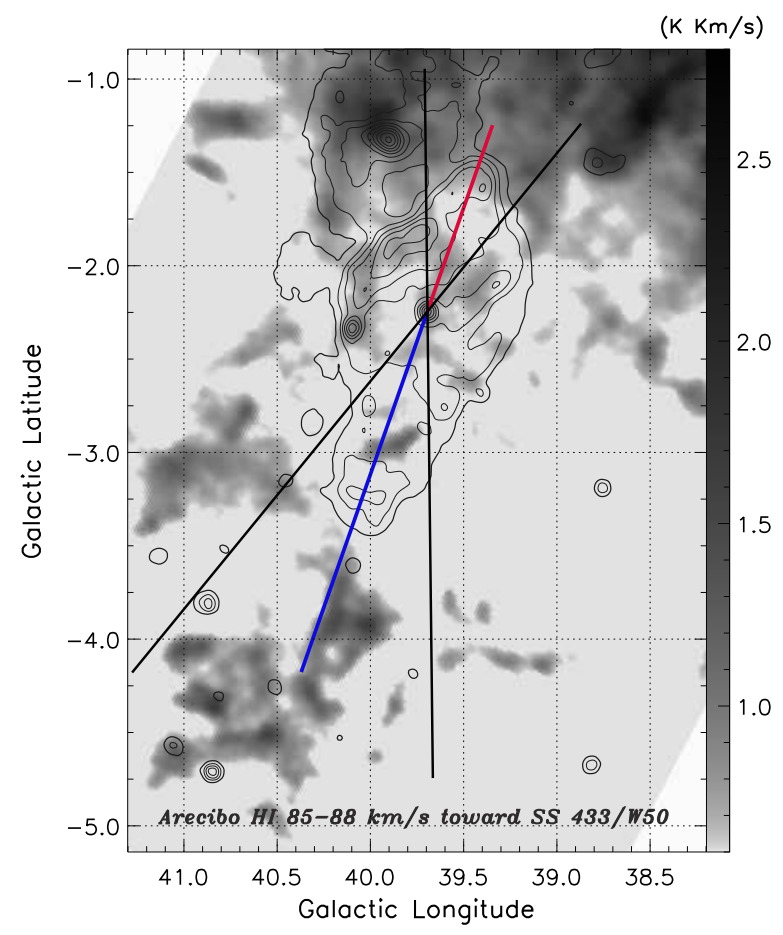

(a)

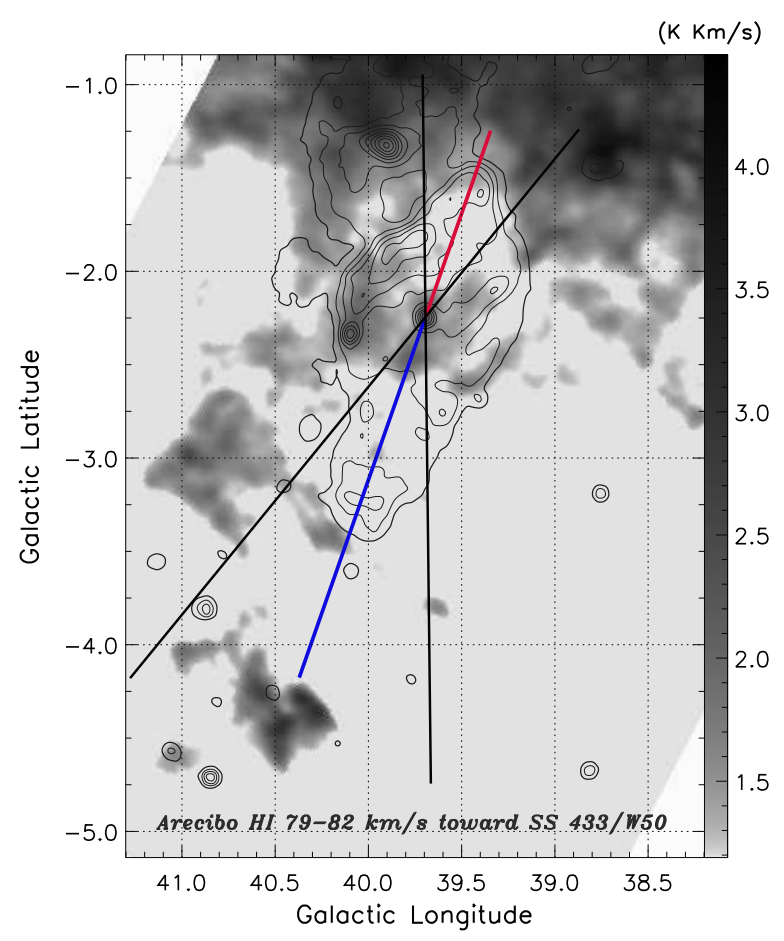

(c)

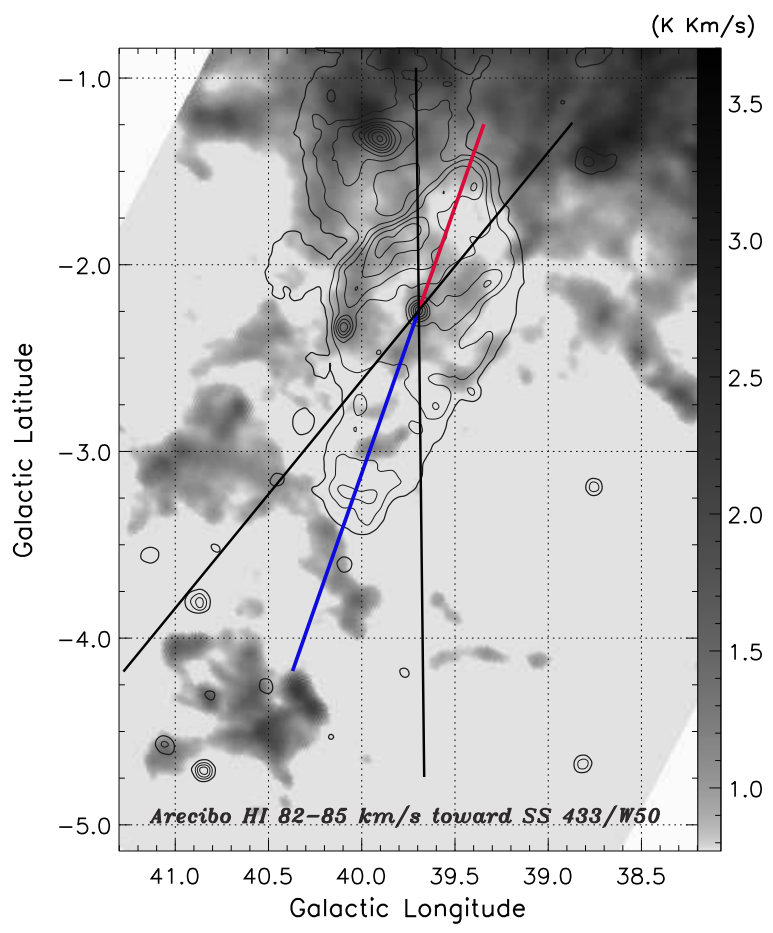

(b)

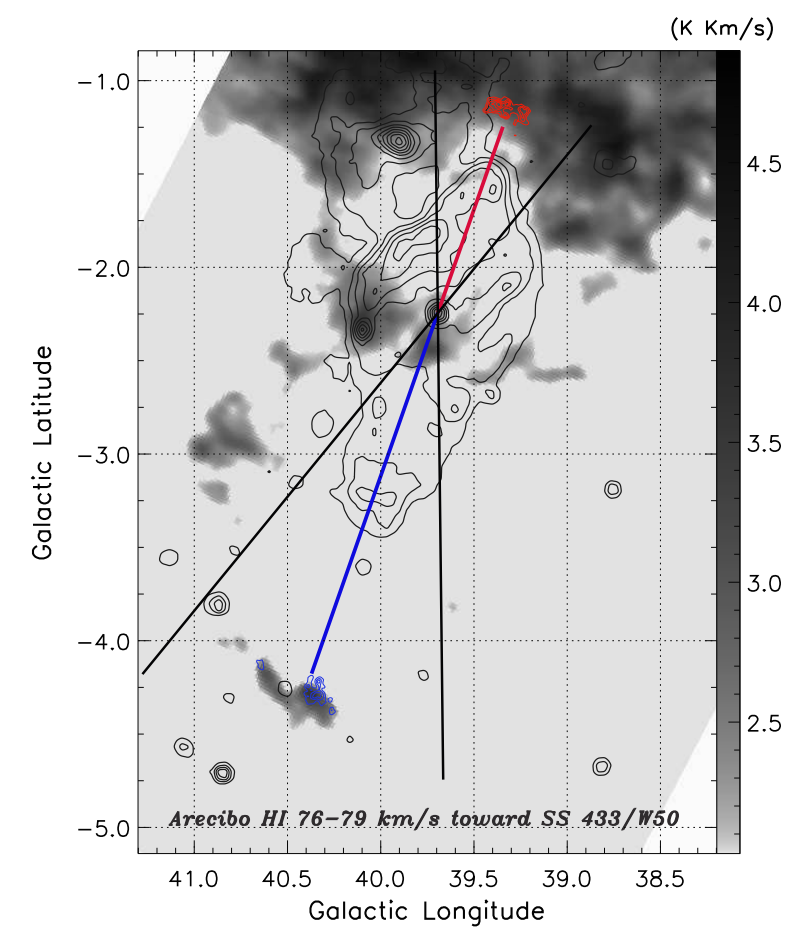

(d)

Figure 11. Arecibo H I emission integrated in the interval of $85-88 \mathrm{~km} \mathrm{~s}^{-1}, 82-85 \mathrm{~km} \mathrm{~s}^{-1}$, 79-82 $\mathrm{km} \mathrm{s}^{-1}$, and 76-79 $\mathrm{km} \mathrm{s}^{-1}$, respectively. All images have been scaled by sin $|b|$ to reduce the $\mathrm{H}$ I emission near the Galactic plane on a large scale and enhance the features far from the Galactic plane. The red and blue line indicates the precession axis of the SS 433 jets. The overlaid black radio contours are the same as in Figure 1 . The red and blue contours are $\mathrm{CO}$ emission from G39.315-1.155 (7.5, 10.0, 12.5, 15.0, and $17.5 \mathrm{~K} \mathrm{~km} \mathrm{~s}^{-1}$ ) and G40.331-4.302 (2.5, 5.0, 7.5, and $\left.10.0 \mathrm{~K} \mathrm{~km} \mathrm{~s}^{-1}\right)$, respectively. 


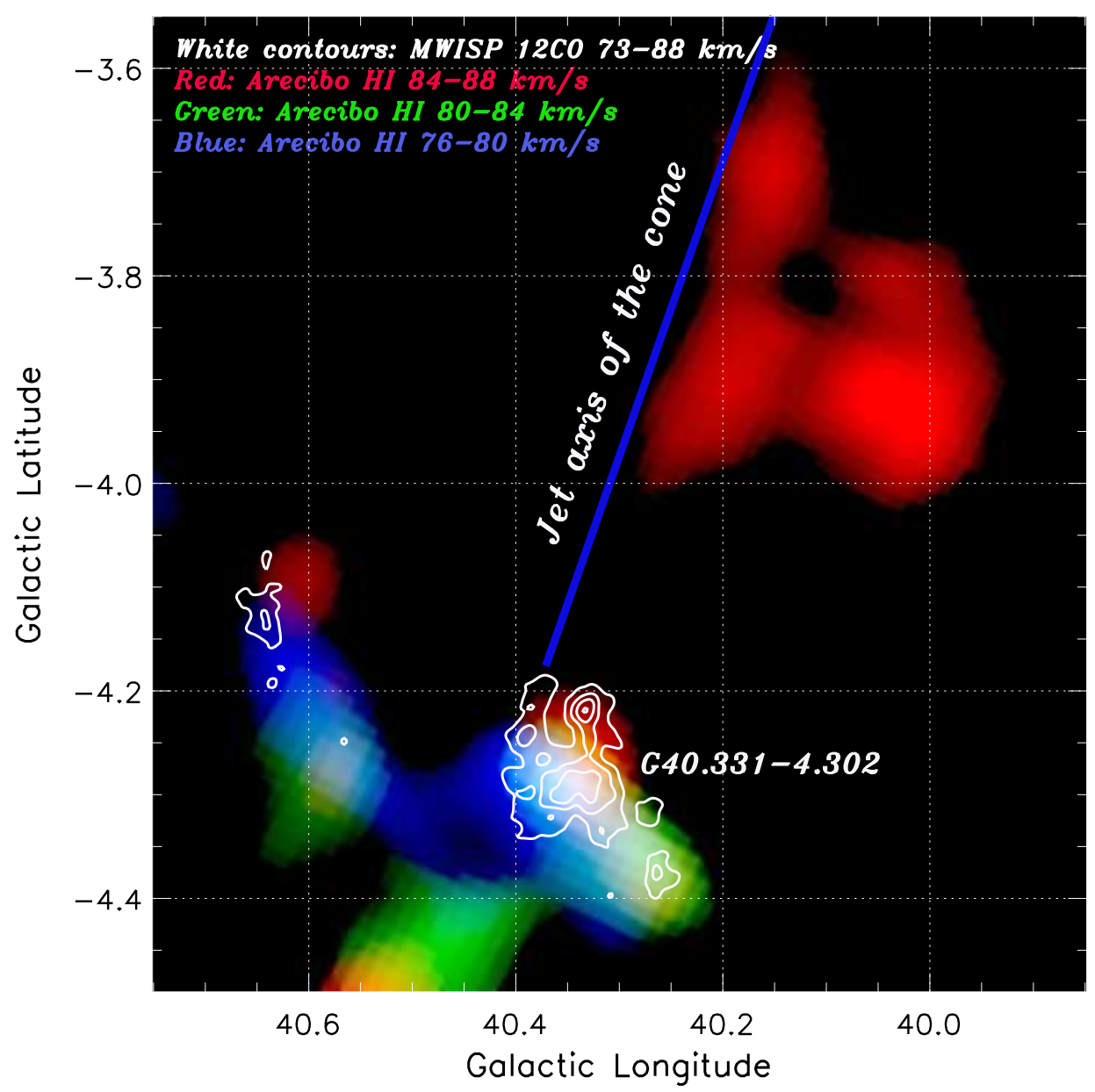

Figure 12. Arecibo H I emission (red: $84-88 \mathrm{~km} \mathrm{~s}^{-1}$, green: $80-84 \mathrm{~km} \mathrm{~s}^{-1}$, and blue: $76-80 \mathrm{~km} \mathrm{~s}^{-1}$ ) toward MC G40.331-4.302. The thick blue line indicates the precession axis of the approaching cone of the SS 433 jets. The CO contour levels start from $2.5 \mathrm{~K} \mathrm{~km} \mathrm{~s}^{-1}$ and increase by a step of $2.5 \mathrm{~K} \mathrm{~km} \mathrm{~s}^{-1}$. 


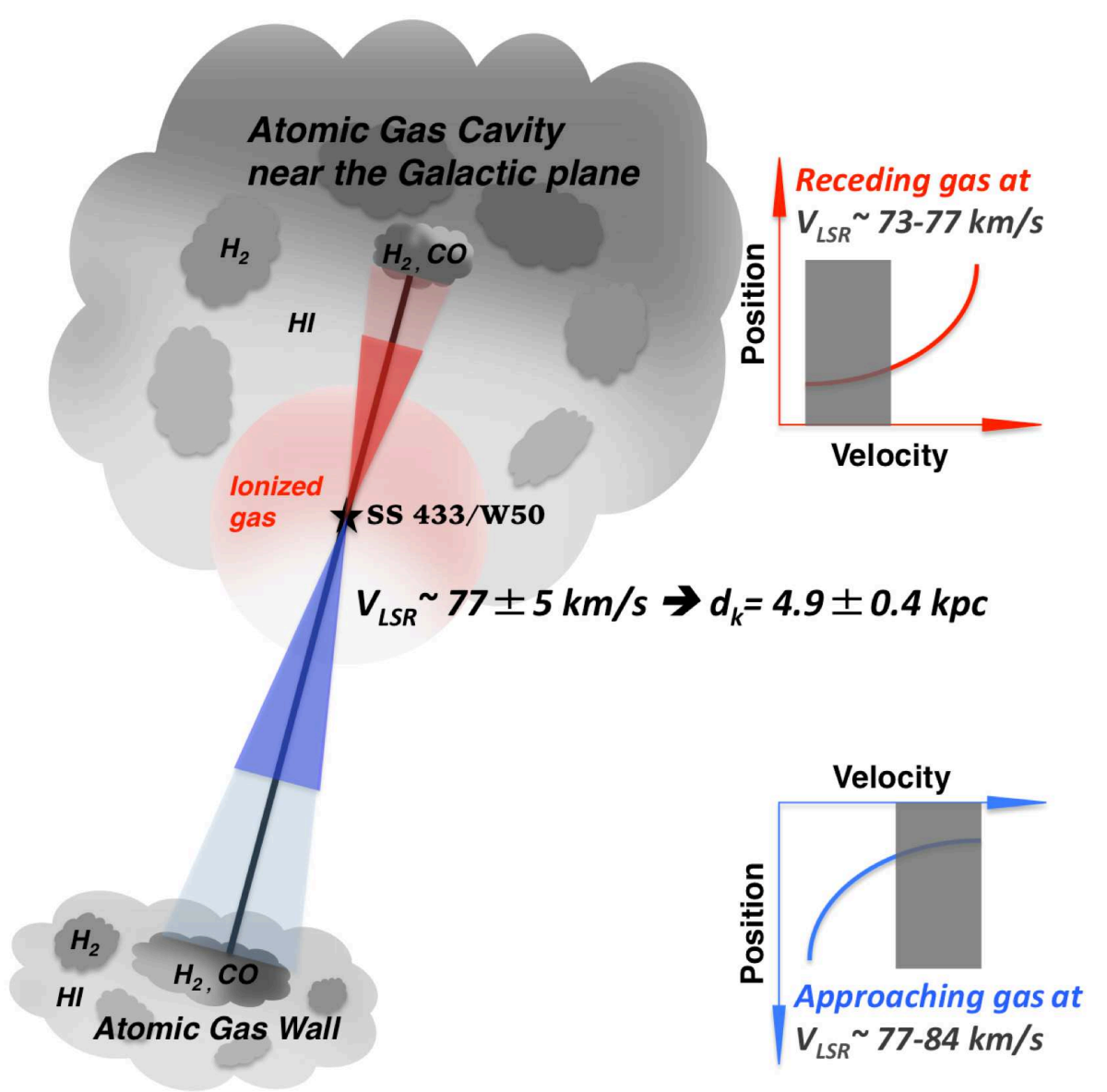

Figure 13. A schematic diagram toward SS 433/W50. The opening angle of the cone is about $\pm 10^{\circ}$ (see Section 3.4) around the precession axis of the SS 433 jets (thick black line). For the right PV diagrams, the shadows represent the LSR velocity of the surrounding gas, while the curves exhibit the velocity changes of the perturbed gas (see Figures 6-7). 